\title{
Incoherent Fermi-Pasta-Ulam Recurrences and Unconstrained Thermalization Mediated by Strong Phase Correlations
}

\author{
M. Guasoni, ${ }^{1,2}$ J. Garnier, ${ }^{3}$ B. Rumpf, ${ }^{4}$ D. Sugny, ${ }^{2,5}$ J. Fatome, ${ }^{2}$ F. Amrani, ${ }^{2}$ G. Millot, ${ }^{2}$ and A. Picozzi ${ }^{2}$ \\ ${ }^{1}$ Optoelectronics Research Centre, University of Southampton, Southampton SO171BJ, United Kingdom \\ ${ }^{2}$ Laboratoire Interdisciplinaire Carnot de Bourgogne (ICB), UMR 6303 CNRS-Université Bourgogne \\ Franche-Comté, F-21078 Dijon, France \\ ${ }^{3}$ Laboratoire de Probabilités et Modèles Aléatoires, University Paris Diderot, \\ 75205 Paris Cedex 13, France \\ ${ }^{4}$ Department of Mathematics, Southern Methodist University, Dallas, Texas 75275, USA \\ ${ }^{5}$ Institute for Advanced Study, Technische Universität München, D-85748 Garching, Germany \\ (Received 31 August 2016; revised manuscript received 28 November 2016; published 6 March 2017)
}

The long-standing and controversial Fermi-Pasta-Ulam problem addresses fundamental issues of statistical physics, and the attempt to resolve the mystery of the recurrences has led to many great discoveries, such as chaos, integrable systems, and soliton theory. From a general perspective, the recurrence is commonly considered as a coherent phase-sensitive effect that originates in the property of integrability of the system. In contrast to this interpretation, we show that convection among a pair of waves is responsible for a new recurrence phenomenon that takes place for strongly incoherent waves far from integrability. We explain the incoherent recurrence by developing a nonequilibrium spatiotemporal kinetic formulation that accounts for the existence of phase correlations among incoherent waves. The theory reveals that the recurrence originates in a novel form of modulational instability, which shows that strongly correlated fluctuations are spontaneously created among the random waves. Contrary to conventional incoherent modulational instabilities, we find that Landau damping can be completely suppressed, which unexpectedly removes the threshold of the instability. Consequently, the recurrence can take place for strongly incoherent waves and is thus characterized by a reduction of nonequilibrium entropy that violates the $H$ theorem of entropy growth. In its long-term evolution, the system enters a secondary turbulent regime characterized by an irreversible process of relaxation to equilibrium. At variance with the expected thermalization described by standard Gibbsian statistical mechanics, our thermalization process is not dictated by the usual constraints of energy and momentum conservation: The inverse temperatures associated with energy and momentum are zero. This unveils a previously unrecognized scenario of unconstrained thermalization, which is relevant to a variety of weakly dispersive wave systems. Our work should stimulate the development of new experiments aimed at observing recurrence behaviors with random waves. From a broader perspective, the spatiotemporal kinetic formulation we develop here paves the way to the study of novel forms of global incoherent collective behaviors in wave turbulence, such as the formation of incoherent breather structures.

DOI: 10.1103/PhysRevX.7.011025

Subject Areas: Nonlinear Dynamics, Statistical Physics

\section{INTRODUCTION}

Recurrence as discovered by Fermi, Pasta, and Ulam is the phenomenon of an apparent reversal of thermalization that occurs in a variety of physical systems [1]. Their numerical study of the dynamics of an anharmonic chain of particles with a single sine wave initial condition shows that energy spreads out from the initially occupied mode to neighboring modes, apparently approaching the thermodynamic equilibrium state of energy equipartition.

Published by the American Physical Society under the terms of the Creative Commons Attribution 3.0 License. Further distribution of this work must maintain attribution to the author(s) and the published article's title, journal citation, and DOI.
Surprisingly, this process is subsequently reversed as the nonlinear dynamics of the chain leads to an energy flow back to the initially occupied mode. Instead of the expected irreversible process of thermalization, the system exhibits recurrent oscillations between a state that is similar to the initial condition and a seemingly disordered state where energy is spread out over several modes.

The paradox of the Fermi-Pasta-Ulam recurrence had a deep impact on the development of nonlinear science [2-6], in particular, in the context of ergodic theory, soliton theory, and integrability [7-15]. Aside from the specific model equation originally considered by Fermi, Pasta, and Ulam [1], the recurrence effect is a general phenomenon found in a variety of almost integrable models whose phase space is strongly segmented by the existence of a large number 
of nearly conserved quantities. As a result, a trajectory emanating from a Benjamin-Feir modulational instability can return nearby the original state, while weak nonintegrable terms cause only a slow irreversible thermalization. The recurrence phenomenon has been studied experimentally in deep water waves [16], magnetic films [17], and optical systems [18-20]. In recent years, recurrence behaviors have attracted an enormous interest for explaining the formation of extreme wave events (rogue or freak waves) in oceans and photonic seas [21,22], in relation to oscillatory breather solutions of integrable nonlinear Schrödinger equations (NLSE) [23-35]. In these cases, recurrent oscillatory behaviors emerge from coherent wave initial conditions, so that one might think that phase coherence constitutes a prerequisite for the recurrence phenomenon. There is an intuitive appeal in the idea that weakly nonlinear random waves should not exhibit reversible recurrences, but instead a monotonic irreversible process of thermalization to equilibrium. Such irreversible processes can be precisely formulated by using the welldeveloped wave turbulence theory [36-41], which has been successfully applied to a huge variety of physical systems $[7-9,11,42-48]$. The wave turbulence theory is formally based on irreversible kinetic equations that describe an irreversible process of thermalization to equilibrium, as expressed by the fundamental $H$ theorem of entropy growth.

In contrast to this common intuitive picture, here, we present and explain the new phenomenon of recurrence of incoherent nonlinear waves in optics, fluids, plasmas, and Bose-Einstein condensates. We show that large convection among a pair of random waves is responsible for a recurrence phenomenon that manifests itself in oscillations similar to the Fermi-Pasta-Ulam recurrence. However, the recurrence effect occurs under radically different physical conditions: Firstly, the recurrence that we observe does not originate in integrability. Secondly, unlike recent studies dealing with purely coherent wave evolutions [20,26-35], here, the recurrence emerges from strongly incoherent random waves. More precisely, the incoherent recurrence is characterized by a reduction of nonequilibrium entropy that violates the $H$ theorem of entropy growth far from integrability.

We explain this phenomenon by showing that convection leads to the spontaneous emergence of strongly correlated fluctuations in the turbulent wave system. For this purpose, we develop a nonequilibrium kinetic theory that accounts for the existence of phase correlations among the random waves. Phase correlations are known to play a role in different cases [7-9,49-58]; in particular, they lead to the formation of large-scale coherent structures (solitons or condensates) emerging from a strongly nonlinear turbulent regime [38-46,59-65] - a coherent structure being inherently a phase-correlated object. Also note that stochastic recurrences of random waves have been predicted recently for water waves governed by Alber's equation when nonlinear effects are stronger than linear dispersive effects [66]. In contrast, here, we consider a system of weakly interacting random waves, in which phase correlations are expected to decay, as precisely described by the wave turbulence theory. At variance with this usual understanding of wave turbulence, our theory reveals that strong phase correlations emerge spontaneously and grow exponentially by virtue of a novel form of modulational instability. More precisely, in contrast to the expected Landau damping that provides a stabilizing effect against the incoherent modulational instability [46,67-72], here, we identify a regime in which Landau damping can be completely suppressed. This has two major consequences. It first explains why our recurrences can take place in the weakly nonlinear regime and thus violate the $H$ theorem of entropy growth. In addition, the suppression of Landau damping makes possible the emergence of strong phase correlations even for highly incoherent waves. Our theory then reveals that the expected natural loss of "phase information" by irreversible processes is partly offset by this spontaneous creation of correlated fluctuations. It turns out that our phase-correlation kinetic theory preserves the time-reversal symmetry and describes in detail the phenomenon of recurrence in a nonintegrable random wave system.

In its long-term evolution, the system enters a secondary turbulent regime that degrades the phase information stored in phase correlations, which reestablishes temporal irreversibility in the system. However, at variance with the expected thermalization and the maximum entropy procedure underlying Gibbsian statistical mechanics [4,73], here, the thermalization process is shown to ignore the usual constraints dictated by energy (Hamiltonian) and momentum conservation. This establishes a novel scenario of thermalization relevant to a large variety of systems, e.g., optical polarization, magnet systems, waves in nonlinear periodic lattices, or the resonant three-wave interaction. This unconstrained thermalization process, as well as the critical phase-correlation effects underlying the incoherent recurrences, constitutes remarkable phenomena that can shed new light on the fundamental origins of irreversibility in turbulent systems operating far from thermodynamic equilibrium.

\section{RECURRENCES FAR FROM INTEGRABILITY}

\section{A. Nonlinear Schrödinger model}

We study the role of convection among two incoherent waves by considering a system of two coupled NLSEs, which is known as a universal model for the description of vector phenomena in nonlinear wave systems. This model describes, for instance, the evolution of the orthogonal polarization components of a light beam that propagates in an optical fiber [74]. It also describes coupled light 
and Langmuir waves in plasma [75], deep ocean waves propagating along two different directions in hydrodynamics [76], systems of coupled electrical oscillators [77], or the matter wave dynamics of binary mixtures of different types of Bose-Einstein condensates [78]. The model can be written in the following form:

$$
\begin{gathered}
i \partial_{t} u=-\partial_{x x} u-i w \partial_{x} u+\left(|u|^{2}+\kappa|v|^{2}\right) u \\
i \partial_{t} v=-\eta \partial_{x x} v+i w \partial_{x} v+\left(|v|^{2}+\kappa|u|^{2}\right) v
\end{gathered}
$$

where $u(x, t)$ and $v(x, t)$ denote the amplitudes of the interacting waves. For convenience, we write the NLSE in dimensionless form; that is, we normalize the problem with respect to the nonlinear time, $\tau_{0}=1 /\left(\gamma_{0} N\right)$, and the "healing" length, $\Lambda=\sqrt{\beta_{u} \tau_{0}}$, where $\gamma_{0}$ is the nonlinear coefficient, $\beta_{u}\left(\beta_{v}\right)$, the dispersion coefficient of $u(v)$, and $N$ is the total energy of the waves. In these units, $\kappa$ denotes the ratio between the cross- and self-interaction coefficients and $\eta=\beta_{v} / \beta_{u}$. Note that the equations are written in a reference frame of average velocity of $u$ and $v$, so that the parameter $w$ denotes the amount of convection in the system. The initial conditions are incoherent waves modeled by random fields that exhibit fluctuations that are statistically homogeneous in space. In the numerical simulations, we consider periodic boundary conditions over the interval $[0, L]$, with $L$ that is much larger than any characteristic length scale of the problem.

The NLSE conserves three important quantities: the partial "energies" $N_{\varphi}=(1 / L) \int_{0}^{L}|\varphi|^{2} d x$, with $\varphi=u, v$, and the Hamiltonian, $\tilde{H}=\tilde{E}+\tilde{U}$, which has a linear contribution

$$
\tilde{E}=\frac{1}{L} \int_{0}^{L} w\left(\tilde{P}_{v}-\tilde{P}_{u}\right)+\left|\partial_{x} u\right|^{2}+\eta\left|\partial_{x} v\right|^{2} d x
$$

where $\tilde{P}_{\varphi}=\operatorname{Im}\left(\varphi \partial_{x} \varphi^{*}\right)$, with $\varphi=u, v$, and a nonlinear contribution

$$
\tilde{U}=\frac{1}{L} \int_{0}^{L} \frac{1}{2}\left(|u|^{4}+|v|^{4}\right)+\kappa|u|^{2}|v|^{2} d x .
$$

Note that the linear contribution to the Hamiltonian that is due to convection (first term in $\tilde{E}$ ) is not positive or negative definite, a feature that will be shown to play a key role in the process of unconstrained thermalization. Of course, the total "energy," $N=N_{u}+N_{v}$, is preserved as well, and we have $N=1$ within our normalization. Equations (1) and (2) are integrable for $\eta=\kappa=1$ ( or $\eta=\kappa=-1$ ), regardless of the parameter $w$ [15]. In the following, we consider the nonintegrable case-in particular, we consider the typical value of $\kappa=2 / 3$, which corresponds to a realistic experimental configuration in an optical fiber system [74].

\section{B. Inapplicability of the wave turbulence theory}

The effect of Fermi-Pasta-Ulam recurrence of purely coherent waves has been widely studied in the framework of NLSE models in relation to the Benjamin-Feir modulational instability $[16,18-20,72]$. As a result of this instability, the initial coherent waves (usually called "pump waves") lead to the amplification of two spectral sidebands [74]. Once the sidebands reach an amplitude comparable to the pump amplitudes, the system enters the nonlinear regime of modulational instability, which is characterized by a reversible transfer of energy from the sidebands to the pumps. This leads to the well-known phenomenon of coherent recurrences mediated by modulational instability $[16,18-20,72]$. In the following, we study the existence of recurrence behaviors by considering initial random waves. We anticipate that the effect of incoherent recurrence we present is also related to a modulational instability, although this instability is of a different nature than the usual incoherent modulational instability [46,67-72].

We consider a highly incoherent (i.e., weakly nonlinear) regime where the strong randomness of the waves makes linear dispersive effects dominant with respect to nonlinear effects, $|\tilde{E}| \gg \tilde{U}$ at $t=0$. The initial incoherent waves have a Gaussian spectrum $\left[\sim \exp \left[-k^{2} /\left(2 \sigma^{2}\right)\right]\right]$ with independent random spectral phases; i.e., they obey Gaussian statistics with a correlation length $\lambda_{c} \sim 1 / \sigma$. The simulations reveal the existence of incoherent recurrences that originate in an instability apparently similar to the modulational instability of fully coherent waves [74]. Hence, we study the incoherent recurrences as in the coherent case and analyze the energy transfer among the incoherent pumps $\left(u_{p}, v_{p}\right)$ and incoherent sideband components $\left(u_{s}, v_{s}\right)$, whose corresponding spectra are centered at the maximum growth rate of the (coherent) modulational instability, i.e., at $k= \pm w$ for $w \gg 1$. We thus split the fields as

$$
\begin{aligned}
& u(x, t)=u_{p}(x, t)+u_{s}(x, t) \exp (-i w x), \\
& v(x, t)=v_{p}(x, t)+v_{s}(x, t) \exp (+i w x),
\end{aligned}
$$

where $w$ is sufficiently large so that the incoherent pump and sideband components have no spectral overlap and can be discerned-we recall that the spectral bandwidth is of the order $\sigma \sim 1 / \lambda_{c} \sim 1$, while $w \gg 1$. The analysis reveals that the pump and sideband components remain discerned throughout the evolution.

We report in Fig. 1(a) the evolution of the energy ratio between a pump and its sideband, $N_{u_{p}}(t) / N_{u}$, where we remind the reader that $N_{u}=N_{u_{p}}+N_{u_{s}}=$ const, with $N_{u_{p}}(t)=(1 / L) \int_{0}^{L}\left|u_{p}\right|^{2} d x$. For moderate convection $w \sim 1$, we recover the expected result where the recurrences are inhibited by the incoherence of the waves. In this case, the system exhibits an irreversible evolution characterized by a monotonous process of entropy production, which is 

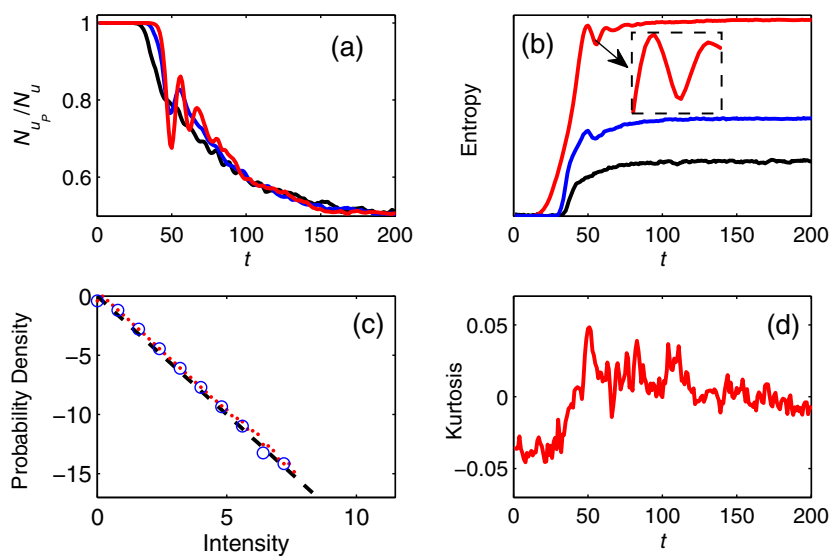

FIG. 1. Incoherent recurrences. Evolutions of the (a) power ratio $N_{u_{p}}(t) / N_{u}$ and (b) total entropy $\tilde{\mathcal{S}}(t)$, for $w=5$ (black line), $w=9$ (blue line), and $w=25$ (red line) from NLSE simulations: The recurrences get more pronounced as $w$ increases, which violates the $H$ theorem of entropy growth $(\sigma=0.47, \eta=1)$. (c) Probability density function (PDF) in logarithmic scale of the intensity $|u|^{2}$ of the wave at $t=50$ (blue circles) and $t=60$ (dashed red line) corresponding to $w=25$ [red lines in (a) and (b)]: Gaussian statistics is preserved throughout incoherent recurrences, as confirmed by the evolution of the kurtosis (d). Note that the dashed dark line in (c) shows the purely exponential law inherent to Gaussian statistics of the wave amplitudes.

illustrated in Fig. 1(b) (black line). This is consistent with the $H$ theorem of entropy growth, $\partial_{t} \tilde{\mathcal{S}} \geq 0$, where the nonequilibrium entropy is defined by $\tilde{\mathcal{S}}=\tilde{\mathcal{S}}_{u}+\tilde{\mathcal{S}}_{v}$, with

$$
\tilde{\mathcal{S}}_{\varphi}(t)=\int \log \left[\tilde{n}_{\varphi}(k, t)\right] d k,
$$

where $\tilde{n}_{\varphi}(k, t)$ are the spectra of the waves $(\varphi=u, v)$ [36,38-40]; see Appendix A.

In contrast to this monotonous behavior, for large convection $w \gg 1$, the energy ratio $N_{u_{p}}(t) / N_{u}$ exhibits recurrences: After some time, the energy in the incoherent sideband is reversibly transferred back to the incoherent pump, as illustrated in Fig. 1(a) (red line). This is characterized by a reduction of nonequilibrium entropy that violates the $H$ theorem; see Fig. 1(b) (red line). The wave turbulence theory cannot explain this recurrence phenomenon, despite the fact that it occurs in the weakly nonlinear regime.

We confirm that the recurrences take place in the weakly nonlinear regime by different methods. We first note that in the simulation reported in Fig. 1, the ratio between nonlinear and linear energies is of the order $\tilde{U} / \tilde{E} \sim 10^{-1}$ throughout the whole evolution $(0<t<200)$. In addition, we analyze separately the contribution to the linear energy that is due to convection $\left(\tilde{E}_{1}\right)$ and the contribution due to dispersion $\left(\tilde{E}_{2}\right)$, with $\tilde{E}=\tilde{E}_{1}+\tilde{E}_{2}$. The analysis reveals that near the recurrence (i.e., for $t \gtrsim 50$ ) the ratio is of the order $\tilde{U} /\left|\tilde{E}_{1,2}\right| \sim 10^{-2}$, which confirms the weakly nonlinear regime of interaction. This weak interaction has also been confirmed by the fact that Gaussian statistics is maintained throughout the evolution. This is illustrated by the analysis of the probability density function (PDF) of the intensity of the waves, which exhibits the purely exponential law inherent to Gaussian statistics, as shown in Fig. 1(c). We also study the evolution of the kurtosis, which is a parameter that measures the deviation from Gaussian statistics $\left(K_{\varphi}=\left\langle|\varphi|^{4}\right\rangle /\left(2\left\langle|\varphi|^{2}\right\rangle^{2}\right)-1\right.$, with $\varphi=u, v)$. As illustrated in Fig. 1(d), the kurtosis remains smaller than a few percent, and thus confirms the Gaussian statistics of the waves throughout the incoherent recurrences.

Note the presence of a peak in the kurtosis at $t=50$, which occurs almost at the same time that the time derivative of the entropy becomes negative, i.e., when the energy in the sideband component $u_{s}$ reaches its maximum. This may be ascribed to a deviation from Gaussianity of $u_{s}$ during its rapid (exponential) amplification from the pumps $\left(u_{p}, v_{p}\right)$, while for large times, Gaussian statistics is restored by the dominant role of dispersion with respect to nonlinear effects [see Fig. 1(d) for $t \gtrsim 60$ ]. Note, however, that the peak in the kurtosis at $t=50$ is too small to significantly affect Gaussian statistics, as confirmed by the corresponding probability density reported for $t=50$ in Fig. 1(c) (blue circles).

\section{Discussion on the integrable limit}

As already mentioned in the Introduction, the FermiPasta-Ulam recurrence is usually considered as a phenomenon that originates in the property of integrability. This statement should be interpreted in the sense that a nonintegrable system, which is "tangent" to an integrable one, behaves as integrable, at least for some amount of time, a property that has been discussed with care in recent works $[10,11]$. However, it is also important to note that, despite common thought, there is no exact result (no theorem) on the connection between recurrence and integrability.

On the other hand, it is well known that the inverse scattering transform allows one to study an integrable system and that it is possible to develop perturbation methods to analyze almost integrable systems. For moderate times, one can then determine the effects of small perturbations on the evolution of an exactly solvable nonlinear evolution equation. It was first done for the Zakahrov-Shabat inverse scattering transform, that can be applied to the scalar NLSE [79]. The Kaup-KarpmanMaslov perturbation scheme was then extended to other systems, such as the (Manakov) vector NLSE [80,81]. In our setting, if $\kappa$ and $\eta$ are close to one, this approach would predict that the NLSE [Eqs. (1) and (2)] departs from integrability after propagation times of the order of $t_{*} \sim 1 / \max \left(|\kappa-1| N,|\eta-1| / \sigma^{2}\right)$. In our case, however, we observe incoherent recurrences even for values of $\kappa$ far 

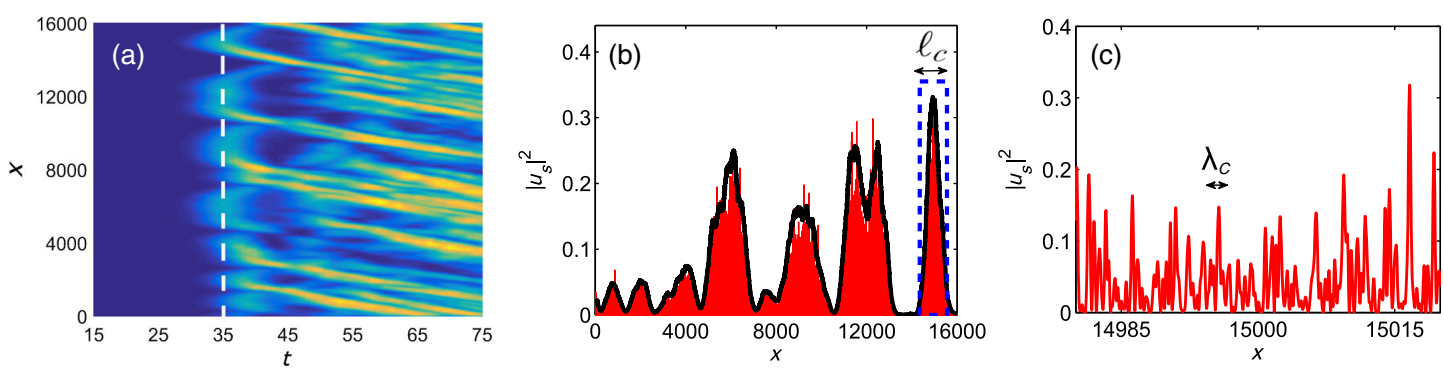

FIG. 2. Microscopic vs macroscopic fluctuations. NLSE simulation showing the spatiotemporal evolution of $\left|u_{s}\right|^{2}(x, t)$ (a) and corresponding spatial profile of the intensity at $t=35$ (b) [see the dashed line in (a)]. Panel (c) shows a zoom on a particular small region within the blue rectangle in (b): Aside from the microscopic fluctuations with correlation length $\lambda_{c} \sim 1$ (c), the waves exhibit macroscopic fluctuations with the large scale $\ell_{c} \sim 4 \pi w / \kappa \simeq 10^{3}$ (b). The envelope of the macroscopic fluctuations is reported by the dark bold line in (b), which denotes the spatial profile of the correlator $n_{u_{s}}(x, t=35)$, whose dynamics is coupled to the other correlators $\left(n_{\varphi_{j}}, m_{\mu}\right)$ by the phase-correlation kinetic equations; see Eqs. (11) and (12).

from one $(\kappa>5)$, which means that the system is far from integrability even for short times.

In addition, by considering the integrable limit $(\eta=\kappa=1)$, the numerical simulations reveal that the

incoherent recurrences disappear without strong convection (for $w \sim 1$ ). This evidences the fact that the incoherent recurrences do not originate in the integrability of the NLSE [Eqs. (1) and (2)].

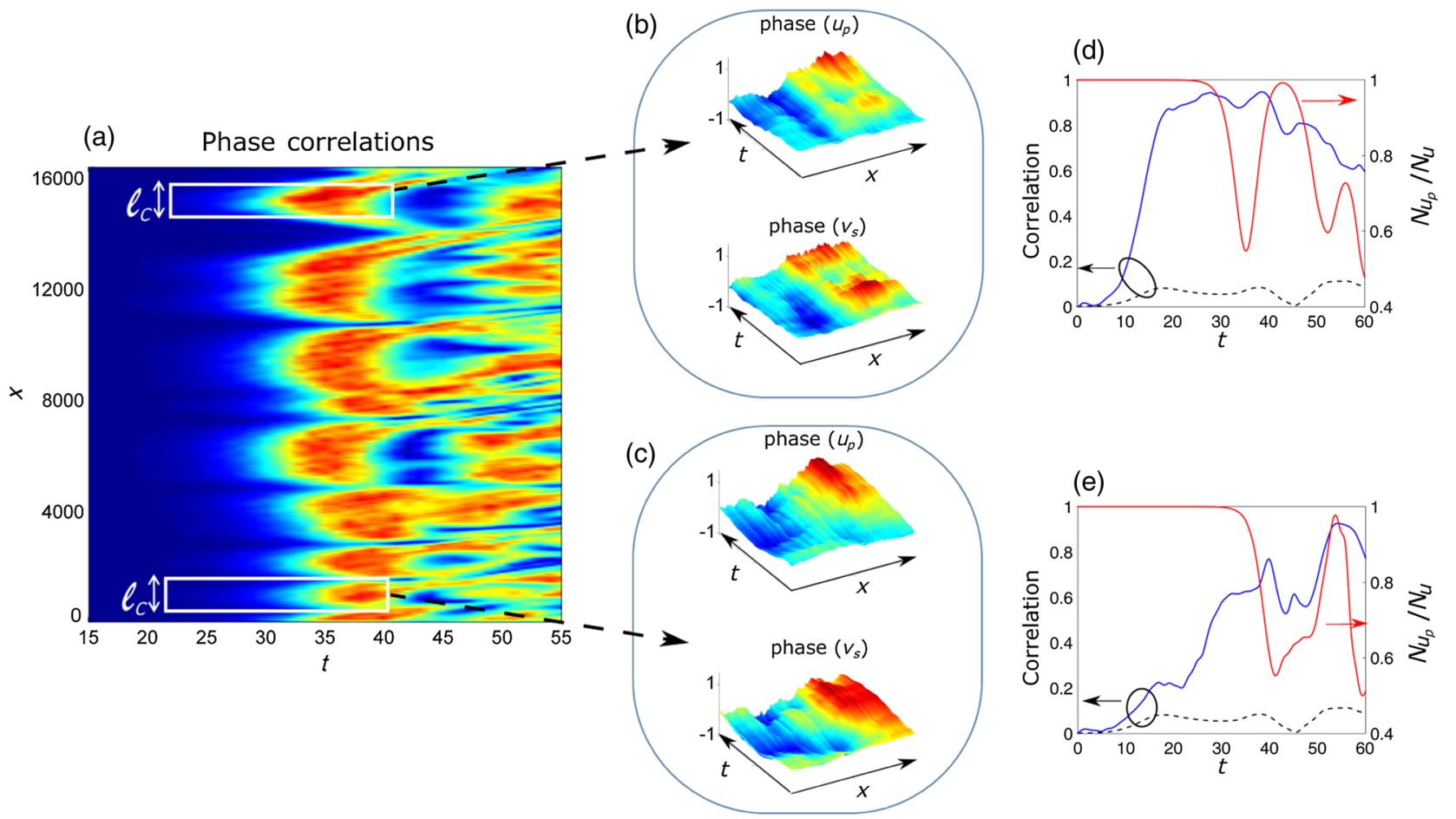

FIG. 3. Creation of local phase correlations. (a) Spatiotemporal evolution of the product $\left|u_{p}(x, t) v_{s}^{*}(x, t)\right|$ obtained from NLSE simulations, showing that phase correlations grow and exhibit a nonhomogeneous statistics. (b), (c) Spatiotemporal evolutions of the phases of the random waves in local spatial regions of size $\ell_{c}$ [white rectangles in (a)]: The similarity of the phases of the random waves $u_{p}$ and $v_{s}$ reflects their strong correlations [note that the space-time windows in the phase plots (b), (c) correspond to those in the white rectangles in (a)]. (d),(e) Evolutions of the normalized phase correlation coefficient $\varrho_{u v}(t)$ given in Eq. (8) (blue line), and power ratio $N_{u_{p}}(t) / N_{u}$ (red line), in local spatial regions [white rectangles in (a)]. Note that there are no correlations between the initial random waves, $\varrho_{u v}(t=0) \simeq 0$ : As a result of the phase-correlation modulational instability [Eq. (16)], the correlation grows up to almost unity, $Q_{u v} \simeq 1$. The dashed dark lines in (d) and (e) show the correlation coefficient $Q_{u v}(t)$ computed over the whole numerical spatial window, showing that there is no global phase correlation, $\varrho_{u v}(t) \lesssim 0.1$ : A complete phase correlation $\left(\varrho_{u v} \simeq 1\right)$ solely emerges in local spatial regions of scale $\ell_{c}$, which in turn leads to almost complete local recurrences, $N_{u_{p}} / N_{u} \sim 1$ [red lines in (d) and (e)]. Parameters are $\sigma=7.5, \eta=1, \kappa=2 / 3, w=25$. 


\section{Creation of correlated fluctuations}

The simulations do not reveal any apparent phase coherence among the random waves, a feature that is confirmed by a global analysis of phase correlations. In order to properly discuss this aspect, we first note that the random waves exhibit a double structure: Aside from the microscopic fluctuations with correlation length $\lambda_{c} \sim 1$, the waves exhibit macroscopic fluctuations with a large length scale $\ell_{c} \sim 10^{3}$. The nonhomogeneous nature of the random waves is illustrated in Fig. 2, which clearly shows the separation of scales between the microscopic and macroscopic fluctuations. The important point to note is that, considering individual local spatial regions of quasihomogeneous statistics with typical size $\ell_{c}$, we find that a strong phase correlation emerges among the random waves. This unexpected result is illustrated in Fig. 3, which reports the global spatiotemporal evolution of $\left|u_{p}(x, t) v_{s}^{*}(x, t)\right|$, as well as the evolutions of the phases in the local spatial regions defined by the length scale $\ell_{c}$; see the two white rectangles in Fig. 3(a). We can notice a remarkable similarity among the phases of the random waves in Figs. 3(b) and 3(c), which reflects the emergence of a strong correlation among them.

We confirm this fact through the analysis of the normalized phase correlation coefficient

$$
\varrho_{u v}(t)=\frac{\left|\left\langle u_{p} v_{s}^{*}\right\rangle(t)\right|}{\left[N_{u_{p}}(t) N_{v_{s}}(t)\right]^{1 / 2}} .
$$

In order to distinguish the local and global phase correlations, we consider two different spatial averages, $\left\langle u_{p} v_{s}^{*}\right\rangle(t)=\left(1 / L_{0}\right) \int_{0}^{L_{0}} u_{p}(x, t) v_{s}^{*}(x, t) d x$ : For the global analysis, the average is taken over the whole numerical window, $L_{0}=L$, while for the local analysis, $L_{0}=\ell_{c}$ denotes the size of the rectangular regions in Fig. 3(a). In contrast to the global analysis [dark dashed line in Figs. 3(d) and 3(e)], in local spatial regions we observe the emergence of a strong phase correlation with $\varrho_{u v} \sim 1$ [blue lines in Figs. 3(d) and 3(e)]. This in turn leads to nearly complete recurrences of the incoherent waves, as illustrated in Figs. 3(d) and 3(e) (red lines), which shows that the energy in the sideband is almost completely transferred back to the pump wave. This study then reveals that the incoherent recurrence phenomenon originates in the spontaneous creation of strong local correlations among the random waves.

\section{PHASE-CORRELATION KINETIC THEORY}

\section{A. Normal correlator and anomalous phase correlator}

We explain the incoherent recurrences by developing a kinetic theory that takes into account nonhomogeneous phase correlations among the random waves. More specifically, the derivation of the kinetic equations follows the general procedure based on the wave turbulence theory: Considering the weakly nonlinear regime, linear dispersion effects dominate nonlinear effects and bring the random waves close to Gaussian statistics, which allows one to derive a set of closed equations for the second-order moments of the fields [36,38-40]. However, at variance with the standard wave turbulence approach that neglects phase correlations, we introduce anomalous phase correlators that account for phase correlations among copropagating random waves - see Appendix B for a detailed derivation of the phase-correlation kinetic equations. Note that anomalous correlators have been introduced to describe parametrically unstable magnet systems [37], or coupled NLSE systems [52]. However, the mechanism of convection induce phase correlations reported here $(w \gg 1)$ has not been discussed in these previous works. In particular, in Ref. [52] a set of equations for the correlations describing a reversible transfer of coherence among the waves was derived. However, such a system consists of a set of ordinary differential equations describing a dynamics of the waves which is uncoupled in both the spatial and frequency domains. Accordingly, the system is inherently unable to describe spatial effects, such as the recurrences mediated by modulational instablity or the unconstrained thermalization process we discuss below. We anticipate that the spatiotemporal kinetic formulation we develop here for the coupled evolutions of the conventional and anomalous phase correlators is found in quantitative agreement with the simulations, without using adjustable parameters.

We define the usual "normal correlators" that denote the spectra of the waves:

$$
n_{\varphi_{j}}(x, k, t)=\int B_{\varphi_{j}}(x, \xi, t) \exp (-i k \xi) d \xi,
$$

where $B_{\varphi_{j}}(x, \xi, t)=\left\langle\varphi_{j}(x+\xi / 2, t) \varphi_{j}^{*}(x-\xi / 2, t)\right\rangle$ are the autocorrelation functions $(\varphi=u, v, j=p, s)$. Note that, because the waves exhibit fluctuations that are not homogeneous in space, the spectra depend on the spatial variable $x$. In addition, we introduce the spectra associated with the "anomalous phase correlators" for the pair of copropagating waves:

$$
m_{\mu}(x, k, t)=\int \Gamma_{\mu}(x, \xi, t) \exp (-i k \xi) d \xi
$$

with $\mu=u v, v u$, where $\Gamma_{u v}(x, \xi, t)=\left\langle u_{p}(x+\xi / 2, t) \times\right.$ $\left.v_{s}^{*}(x-\xi / 2, t)\right\rangle \quad$ and $\quad \Gamma_{v u}(x, \xi, t)=\left\langle u_{s}(x+\xi / 2, t) \times\right.$ $\left.v_{p}^{*}(x-\xi / 2, t)\right\rangle$. Performing a multiscale expansion with the small parameter $1 / w \ll 1$, we derive in Appendix B the following phase-correlation kinetic equations:

$$
\begin{gathered}
\left(\partial_{t}+w \partial_{x}\right) n_{u_{p}}(x, k, t)=\mathcal{G}_{n}\left[m_{\mu}\right], \\
i\left(\partial_{t}-w \partial_{x}\right) m_{v u}(x, k, t)=\mathcal{G}_{m}\left[n_{\varphi_{j}}, m_{\mu}\right],
\end{gathered}
$$


while the evolutions of the sideband spectra are deduced from those of the pumps [e.g., $\left.n_{u_{s}}(x, k, t)=n_{v_{p}}(k, t=0)-n_{v_{p}}(x, k, t)\right]$. The functionals $\operatorname{read} \mathcal{G}_{n}\left[m_{\mu}\right]=-2 \kappa \operatorname{Im}\left\{m_{u v}(x, k, t)\left[m_{u v}^{0 *}(x, t)+m_{v u}^{0 *}(x, t)\right]\right\}$ and $\mathcal{G}_{m}\left[n_{\varphi_{j}}, m_{\mu}\right]=-2 m_{v u}(x, k, t)\left\{(2-\kappa)\left[n_{v_{p}}^{0}(x, t)-n_{u_{p}}^{0}(x, t)\right]+\right.$ $\left.(1-\kappa / 2)\left(N_{u}-N_{v}\right)\right\}-\kappa\left[m_{v u}^{0}(x, t)+m_{u v}^{0}(x, t)\right]\left[n_{v_{p}}(k, t=0)-\right.$ $\left.2 n_{v_{p}}(x, k, t)\right]+(1-\eta) k^{2} m_{v u}(x, k, t)$, where we define

$$
\begin{aligned}
& n_{\varphi_{j}}^{0}(x, t)=B_{\varphi_{j}}(x, \xi=0, t), \\
& m_{\mu}^{0}(x, t)=\Gamma_{\mu}(x, \xi=0, t) .
\end{aligned}
$$

The corresponding equations for $n_{v_{p}}(x, k, t)$ and $m_{u v}(x, k, t)$ can be deduced from Eqs. (11) and (12) through a simple substitution; see Appendix B. It is important to note that, in contrast to the wave turbulence formalism (see Appendix A), the phase-correlation kinetic equations (11) and (12) are formally reversible in time.

\section{B. Phase-correlation modulational instability 1. Incoherent modulational instability}

Here, we show that the linearized phase-correlation kinetic equations (11) and (12) predict a modulational instability characterized by an exponential growth of phase correlations. It is important to discuss this phenomenon within the general context of the modulational instability of random waves. The "incoherent modulational instability" refers to a phenomenon in which a statistical homogeneous random wave can become unstable with respect to the growth of weak statistical inhomogeneities, thus leading to a periodically modulated pattern of the random wave. This phenomenon is fundamental and has been widely studied in different contexts [46,67-72,82-86]. At variance with the (Benjamin-Feir) modulational instability of purely coherent waves, the incoherent modulational instability is characterized by a threshold: The instability is suppressed in the weakly nonlinear regime when the incoherence of the waves is increased beyond some critical value. This property is due to a Landau damping, which has a stabilizing effect that tends to suppress the modulational instability [46,67-70,72]. This stabilizing effect is not an ordinary dissipative damping, but an energy-conserving effect that created its own share of confusion about 40 years ago [67]. It is interesting to remark that in the nonlinear stage of the incoherent modulational instability, the system can exhibit recurrence behaviors that can be viewed as the stochastic counterpart of the Fermi-Pasta-Ulam recurrence, a property discussed in detail in the framework of Alber's equation [66]. Note that such recurrences do not occur for strongly incoherent waves, because in the weakly nonlinear regime, the modulational instability is suppressed by the Landau damping - the development of the instability requires that nonlinear effects are stronger than linear dispersion effects (i.e., a Benjamin-Feir index, BFI $\geq 1$, in one spatial dimension [71,72]).

\section{Suppression of Landau damping}

Here, we present a phenomenon of incoherent modulational instability, which is of a different nature than that discussed above [46,67-72,82-86]. First of all, the modulational instability occurs solely for the phase correlations $m_{\mu}(x, k, t)$, so that the two sidebands $\left(u_{s}, v_{s}\right)$ are stable and do not grow. Furthermore, we identify a regime in which Landau damping is completely suppressed, so that the modulational instability does not exhibit any threshold: The phase correlations grow efficiently even for strongly incoherent waves. At variance with the stochastic recurrences predicted in Ref. [66], here, the suppression of Landau damping allows the recurrences to take place in the weakly nonlinear regime, which leads to a violation of the $H$ theorem of entropy growth.

We start to analyze Eqs. (11) and (12) by considering the initial regime of interaction in which phase correlations are negligible and the energy in the sidebands is much smaller than in the pumps $\left(n_{\varphi_{p}} \gg n_{\varphi_{s}}, n_{\varphi_{p}} \gg\left|m_{\mu}\right|\right.$ for small interaction times, $t \lesssim 20$ ). The linearized equations (11) and (12) can be solved by the Fourier-Laplace technique, $\quad \hat{m}_{\mu}(p, k, \lambda)=\int_{0}^{\infty} d t \int d x m_{\mu}(x, k, t) \exp (-\lambda t-i p x)$, which gives the growth rate $\lambda$ of the phase-correlation modulational instability:

$$
1=\frac{i \kappa}{2 \pi} \int\left(\frac{n_{u_{p}}(k, t=0)}{\lambda-i \tilde{\Delta}_{-}(p, k)}-\frac{n_{v_{p}}(k, t=0)}{\lambda-i \tilde{\Delta}_{+}(p, k)}\right) d k,
$$

where $\quad \tilde{\Delta}_{ \pm}(p, k)=(\eta-1) k^{2} \pm p w-2 \Delta$, with $\quad \Delta=$ $\left(N_{u}-N_{v}\right)(1-\kappa / 2)$. As illustrated in Fig. 4(a), Eq. (15) reveals that the incoherence of the pumps significantly reduces the growth rate of the phase-correlation instability. This property is due to a Landau damping effect, which is known to introduce a threshold in the modulational instability of random waves [46,67-72]. However, the unexpected result revealed by Eq. (15) is that such effective damping is completely suppressed for $\eta=1$, so that a strong phase correlation emerges in the system regardless of the amount of pump incoherence. In this case, the phasecorrelation growth rate reads

$$
\lambda_{1}(p)=\sqrt{\kappa^{2} / 4-(w p-\kappa / 2)^{2}},
$$

where we consider, for simplicity, the case where the two pump waves have the same initial spectra $\left[n_{v_{p}}(k, t=0)=n_{u_{p}}(k, t=0)\right]$.

In this instability, the sideband components $\left(u_{s}, v_{s}\right)$ are linearly stable: Their amplifications are delayed by a significant time [see Fig. 4(b), $\tau_{d} \simeq 13$ ] because their growths are driven by the phase correlations $\left[m_{\mu}(x, t)\right]$ in the subsequent nonlinear stage of the phase-correlation 

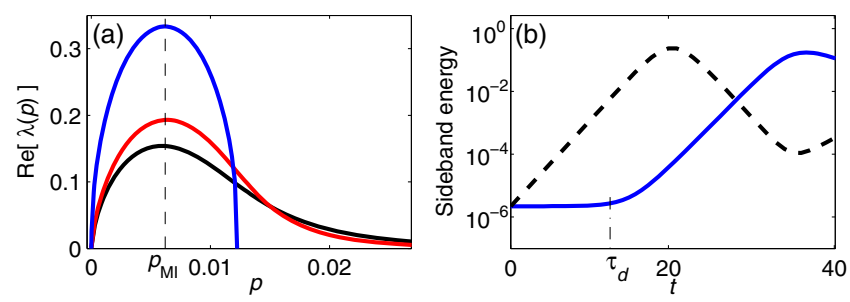

FIG. 4. Phase-correlation modulational instability. (a) Growth rate of phase-correlation instability $\operatorname{Re}[\lambda(p)]$ from Eq. (15) for $\eta=1$ (blue line), $\eta=1.01, \sigma=7.5$ (black line), $\eta=1.1, \sigma=2$ (red line): Landau damping is suppressed for $\eta=1$, thus leading to an efficient phase-correlation growth. The length scale of macroscopic (nonhomogeneous) fluctuations is $\ell_{c} \simeq 2 \pi / p_{\mathrm{MI}}$; see Fig. 2 and Eq. (17). (b) Temporal evolution of the energy in the sideband $\left(u_{s}\right)$ in the presence of coherent (dashed black line) and incoherent (solid blue line) pump waves. When the pump waves $\left(u_{p}, v_{p}\right)$ are incoherent, the phase correlations are unstable, while the sideband components are stable, so that the growth of $u_{s}$ is delayed by a significant time, $\tau_{d} \simeq 13$ (solid blue line). In contrast, when the pump waves are coherent, the standard modulational instability leads to an instantaneous growth of the sideband $u_{s}$ (dashed black line).

instability. Such a time delay simply results from Eq. (11): the sideband growths can only start once the nonlinear product $m_{u v}^{0}(x, t) m_{v u}^{0 *}(x, t)$ reaches the sidebands' noise level.

The phase-correlation instability explains the origin of the macroscopic nonhomogeneous fluctuations of the waves observed in the NLSE simulations in Figs. 2 and 3. This is simply due to the fact that the homogeneous mode of the instability has zero growth rate, $\lambda_{1}(p=0)=0$; see Fig. 4(a). Note that in this way the incoherent recurrences cannot take place homogeneously in space. More precisely, the maximum growth rate in Eq. (16) occurs at $p_{\mathrm{MI}}=\kappa /(2 w)$, so that the large length scale of macroscopic (nonhomogeneous) fluctuations of the waves is

$$
\ell_{c} \simeq 2 \pi / p_{\mathrm{MI}}=4 \pi w / \kappa .
$$

Note that since the interaction coefficient is typically of order $1, \kappa \sim 1$, then $\ell_{c} \sim w \gg 1$. The separation of scales between microscopic fluctuations (with correlation length $\lambda_{c} \sim 1$ ) and macroscopic fluctuations (with $\ell_{c} \sim 10^{3}$ ) is clearly visible in the NLSE simulations, as discussed in Figs. 2 and 3.

\section{Nonlinear stage of phase-correlation modulational instability}

\section{Reduced form of phase-correlation kinetic equations}

The incoherent recurrences take place in the nonlinear stage of the phase-correlation modulational instability, as discussed above through Figs. 1-3. To describe this phenomenon, we remark the important fact that for $\eta=1$, Eqs. (11) and (12) can be simplified by factorizing the correlators into their spatial and spectral contributions. Specifically, Eqs. (11) and (12) admit the following exact solutions:

$$
n_{\varphi_{p}}(x, k, t)=n_{\varphi_{p}}(k, t=0) n_{\varphi_{p}}^{0}(x, t) / N_{\varphi},
$$

for $\varphi=u, v$, and

$$
m_{\mu}(x, k, t)=n_{\varphi_{p}}(k, t=0) m_{\mu}^{0}(x, t) / N_{\varphi},
$$

for $\mu=u v, \varphi=u$ (or $\mu=v u, \varphi=v$ ). Note that this form of the solutions has a simple meaning, namely, that the shapes of the (averaged) spectral profiles of the waves $\left(u_{\varphi_{j}}, v_{\varphi_{j}}\right)$ are preserved during their evolutions. Accordingly, Eqs. (11) and (12) recover the simplified closed form:

$$
\begin{aligned}
\left(\partial_{t}+w \partial_{x}\right) n_{u_{p}}^{0}(x, t) & =\mathcal{G}_{n}^{0}\left[n_{\varphi}^{0}, m_{\mu}^{0}\right], \\
i\left(\partial_{t}-w \partial_{x}\right) m_{v u}^{0}(x, t) & =\mathcal{G}_{m}^{0}\left[n_{\varphi}^{0}, m_{\mu}^{0}\right],
\end{aligned}
$$

and two similar equations for $n_{v_{p}}^{0}(x, t)$ and $m_{u v}^{0}(x, t)$, while $n_{\varphi_{s}}^{0}(x, t)=N_{\varphi}-n_{\varphi_{p}}^{0}(x, t)(\varphi=u, v)$. The functionals read $\mathcal{G}_{n}^{0}\left[n_{\varphi}^{0}, m_{\mu}^{0}\right]=-2 \kappa \operatorname{Im}\left[m_{u v}^{0}(x, t) m_{v u}^{0 *}(x, t)\right]$ and $\mathcal{G}_{m}^{0}\left[n_{\varphi}^{0}, m_{\mu}^{0}\right]=$ $-m_{v u}^{0}(x)\left\{\left(2(\kappa-1)\left[N_{v}-2 n_{v_{p}}^{0}(x)\right]+(2-\kappa)\left[N_{u}-2 n_{u_{p}}^{0}(x)\right]\right\}-\right.$ $\kappa m_{u v}^{0}(x)\left[N_{v}-2 n_{v_{p}}^{0}(x)\right]$. The reduced phase-correlation kinetic Eqs. (20) and (21) provide an accurate description of the incoherent recurrences and their spatial nonhomogeneous nature, as revealed by a remarkable agreement between the kinetic equations (20) and (21) and the NLSE. This is illustrated in Figs. 5(c) and 5(d), which report a direct comparison of the evolutions of the normal and anomalous correlators $\left(n_{\varphi_{j}}^{0}, m_{\mu}^{0}\right)$, obtained by simulations of the NLSE and the kinetic equations (20) and (21). Note that this good agreement is obtained without using adjustable parameters.

\section{Poincaré-Stokes variables: Relevance and limits}

It is interesting to note that the reduced phase-correlation kinetic equations (20) and (21) can be written in a compact form by using the Poincaré-Stokes variables. The Stokes vectors are defined by

$$
\begin{gathered}
\boldsymbol{U}=2\left\{\operatorname{Im}\left(m_{u v}^{0}\right),-\tilde{n}_{u_{p}}^{0}, \operatorname{Re}\left(m_{u v}^{0}\right)\right\}, \\
\boldsymbol{V}=2\left\{\operatorname{Im}\left(m_{v u}^{0}\right), \tilde{n}_{v_{p}}^{0}, \operatorname{Re}\left(m_{v u}^{0}\right)\right\}
\end{gathered}
$$

where $\tilde{n}_{\varphi_{p}}^{0}(x, t)=N_{\varphi} / 2-n_{\varphi_{p}}^{0}(x, t)(\varphi=u, v)$, and evolve on the surface of two spheres of radii $U_{0}^{2}=\sum_{i=1}^{3} U_{i}^{2}=N_{u}^{2}$ and $V_{0}^{2}=\sum_{i=1}^{3} V_{i}^{2}=N_{v}^{2}$. The phase-correlation kinetic equations (20) and (21) can then be recast in the following compact form: 

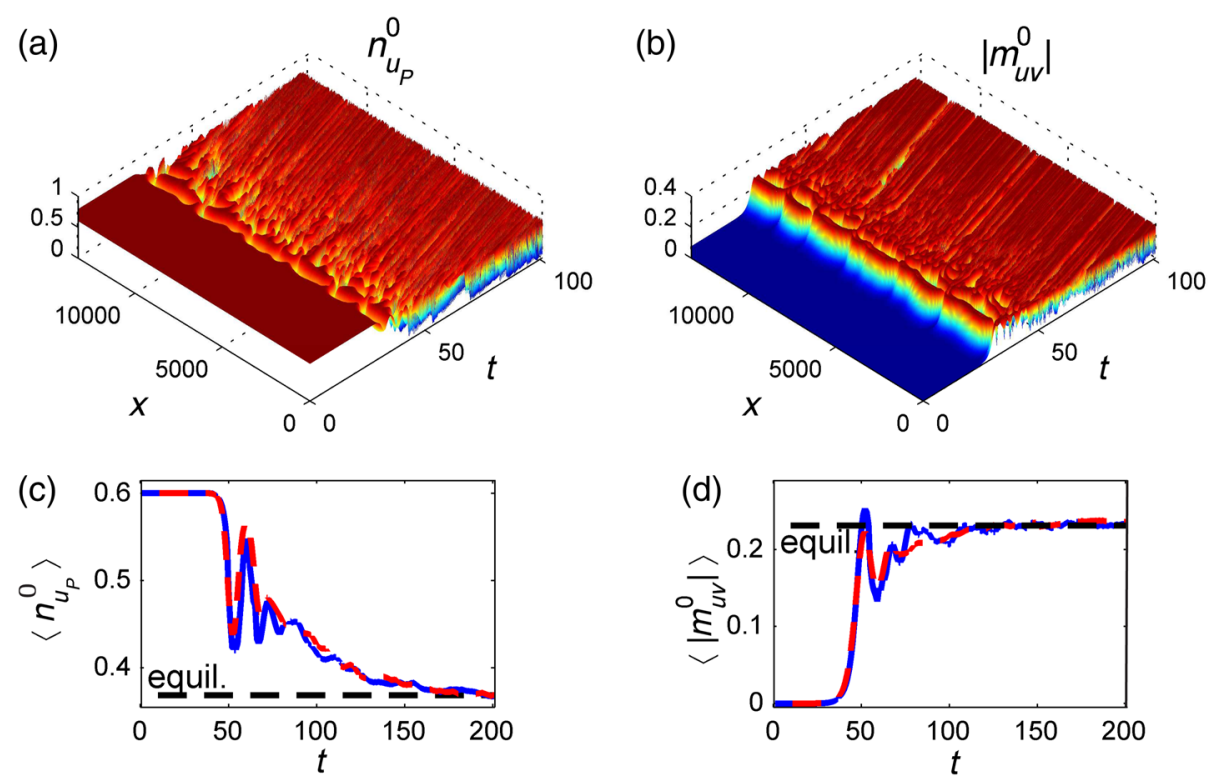

FIG. 5. Long-term evolution: Secondary turbulent regime and unconstrained thermalization. (a),(b) Simulations of the phasecorrelation kinetic equations (20) and (21) showing the evolutions of the normal correlator $n_{u_{p}}^{0}$ (a) and anomalous phase correlator $\left|m_{u v}^{0}\right|$ (b): After a few incoherent recurrences $(t \gtrsim 80)$, the correlators enter a secondary turbulent regime. (d), (e) Corresponding evolutions of the spatial averages of the correlators $\left\langle n_{u_{p}}^{0}\right\rangle(t)$ (c) and $\left\langle\left|m_{u v}^{0}\right|\right\rangle(t)$ (d), obtained from the simulations of the phase-correlation kinetic equations (20) and (21) (dashed red line) and NLSE (blue line): For large times $(t \gtrsim 150)$, the fields relax to the equilibrium state predicted by the theory (horizontal black dashed lines); see Eqs. (35) and (36). Parameters are $N_{u}=0.6, N_{v}=0.4, \kappa=2 / 3, w=54$. Unexpectedly, this equilibrium state is not constrained by Hamiltonian and momentum conservation (see Sec. IV).

$$
\begin{aligned}
& \left(\partial_{t}+w \partial_{x}\right) \boldsymbol{U}=\boldsymbol{U} \times \mathcal{I}(\boldsymbol{U}+\boldsymbol{V}), \\
& \left(\partial_{t}-w \partial_{x}\right) \boldsymbol{V}=\boldsymbol{V} \times \mathcal{I}(\boldsymbol{V}+\boldsymbol{U}),
\end{aligned}
$$

with the diagonal matrix $\mathcal{I}=\operatorname{diag}(\kappa, 2-\kappa, \kappa)$. This formulation unveils the Hamiltonian structure of the phase-correlation kinetic equations; see Appendix C. The Hamiltonian can be split as follows:

$$
\mathcal{H}=\mathcal{H}_{U}+\mathcal{H}_{V}+\mathcal{H}_{\text {int }},
$$

where the cross-interaction term is $\mathcal{H}_{\text {int }}=-\int \boldsymbol{V} \cdot \mathcal{I} \boldsymbol{U} d x$, while the self-interaction contributions read: $\mathcal{H}_{j}=\mathcal{E}_{j}+\mathcal{U}_{j}$, $j=U, V$, with $\mathcal{U}_{U}=-\frac{1}{2} \int \boldsymbol{U} \cdot \mathcal{I} \boldsymbol{U} d x, \mathcal{U}_{V}=-\frac{1}{2} \int \boldsymbol{V} \cdot \mathcal{I} \boldsymbol{V} d x$, $\mathcal{E}_{U}=-w \mathcal{P}_{U}, \mathcal{E}_{V}=w \mathcal{P}_{V}$, and $\mathcal{P}_{j}=\int P_{j} d x, j=U, V$. In addition, Eqs. (24) and (25) conserve the momentum,

$$
\mathcal{P}=\int P_{U}+P_{V} d x
$$

as well as the "magnetization,"

$$
\mathcal{M}=-\frac{1}{2} \int U_{2}+V_{2} d x
$$

as discussed in detail in Appendix C.

The Poincaré-Stokes formalism proved efficient to describe nonlinear polarization phenomena by considering essentially coherent optical waves; see, for instance,
Refs. [87-89]. However, it is important to note that, here, the application of the Poincaré-Stokes formalism exhibits some important differences with respect to the usual approaches. (i) The Stokes vector has no physical interpretation in terms of polarization effects, because the Stokes variables do not refer to a single wave component, but involve a cross-correlation among different components [e.g., $u_{p}(x, t)$ and $v_{s}(x, t)$ for $\boldsymbol{U}$ ]. (ii) In contrast to usual approaches, here, the Stokes vector denotes the averaged envelopes that describe the macroscopic nonhomogeneous fluctuations of the turbulent waves. (iii) The Stokes formalism [Eqs. (24) and (25)] is valid only for $\eta=1$ and a specific initial condition of the form $\boldsymbol{U}(x, t=0)=$ $\left(0, U_{2}, 0\right), \boldsymbol{V}(x, t=0)=\left(0, V_{2}, 0\right)$, where $U_{2}(x, t=0)=U_{0}$ and $V_{2}(x, t=0)=V_{0}$ are constant. For general initial conditions, we deal with eight coupled real equations for $\left(n_{\varphi}, m_{\mu}\right)$, which makes the Stokes parameters irrelevant to our problem. In spite of these limits, we show that the Stokes formulation proves convenient to discuss the thermalization process presented in the following section.

\section{UNCONSTRAINED THERMALIZATION}

\section{A. Secondary turbulent dynamics for the correlators}

The simulations of the phase-correlation kinetic equations (20) and (21) reveal that, for long interaction times, the system enters a second stage characterized by an irreversible evolution toward equilibrium, as illustrated in 
Figs. 5(a) and 5(b) for $t \gtrsim 80$. In this regime, the averaged envelopes $\left[n_{\varphi_{j}}^{0}(x, t)\right]$ that describe the nonhomogeneous fluctuations of the waves themselves undergo strong turbulent fluctuations. This secondary turbulent regime deteriorates the phase information stored in the phase correlations $m_{\mu}^{0}(x, t)$, which have been shown to be responsible for the incoherent recurrences. It turns out that, despite the formal reversibility of Eqs. (20) and (21), the system exhibits an irreversible process of relaxation to equilibrium.

We describe this second turbulent stage of the system by developing a secondary statistical analysis on the equations that rule the evolution of the correlators $\left(n_{\varphi_{j}}^{0}, m_{\mu}^{0}\right)$. In this respect, it is important to note that the phase-correlation kinetic equations (20) and (21) do not exhibit quadratic dispersion relations; i.e., they do not exhibit second-order spatial derivatives. This means that the system [Eqs. (20) and (21)] is weakly dispersive, with purely linear acousticlike dispersion relations. In this way, the highly nonlinear turbulent regime illustrated in Fig. 5 cannot be described by a weakly nonlinear approach, such as the wave turbulence theory. The nonlinear character of this secondary turbulent regime will be confirmed by the probability density functions of the correlators $\left(n_{\varphi_{j}}^{0}, m_{\mu}^{0}\right)$, which will be shown to be of different nature than Gaussian statistics. Note that secondary statistical analysis of a primary mean-field approximation of the problem has been considered in magnet systems under parametric excitation [37].

\section{B. Gibbsian statistical mechanics on the correlators}

We study the equilibrium properties of the phasecorrelation kinetic equations (20) and (21) by following a statistical mechanics approach based on the maximization of the Gibbs entropy, i.e., a functional $\mathcal{S}[\rho]=-\int \rho \log (\rho) d \boldsymbol{K}$ of the PDF $\rho(\boldsymbol{K})$ that operates over the whole phase space, as discussed in Appendix D. For this purpose, it proves convenient to make use of the phase-correlation kinetic equations written in terms of the Poincaré-Stokes variables [Eqs. (24) and (25)], which have been shown to conserve the Hamiltonian $\mathcal{H}$, the momentum $\mathcal{P}$, and the "magnetization" $\mathcal{M}$. According to standard statistical mechanics and information theoretic principles, the equilibrium PDF is obtained by maximizing $\mathcal{S}[\rho]$ under the constraints that the phase-space evolution takes place on the shell that conserves $\mathcal{H}, \mathcal{P}$, and $\mathcal{M}$,

$$
\rho \sim \exp (-\beta \mathcal{H}-\nu \mathcal{P}-\gamma \mathcal{M}),
$$

where $(\beta, \nu, \gamma)$ are the inverse temperatures (Lagrange multipliers) associated to $(\mathcal{H}, \mathcal{P}, \mathcal{M})$ given in Eqs. (26)-(28) $[4,73]$.

\section{Zero inverse temperatures}

In this section, we show that the statistical mechanics approach of the phase-correlation kinetic equations (20) and (21) [or Eqs. (24) and (25)] reveals the existence of an irreversible process of thermalization that is quite general, in the sense that it is relevant to a large class of weakly dispersive wave systems, as we discuss below in more detail (see Sec. VA). Such a thermalization process is unconstrained in the sense that it is not dictated by the usual laws of energy and momentum conservation; i.e., the equilibrium state is characterized by zero inverse temperatures:

$$
\nu=\partial \mathcal{S} / \partial \mathcal{P}=0, \quad \beta=\partial \mathcal{S} / \partial \mathcal{H}=0 .
$$

To avoid confusion, we clarify that this process of unconstrained thermalization does not occur for the microscopic fluctuations of the random waves $(u, v)$ (with correlation length $\lambda_{c} \sim 1$ ), but for their macroscopic nonhomogeneous fluctuations $\left(n_{\varphi_{j}}, m_{\mu}\right)$ (with correlation length $\ell_{c} \sim w \gg 1$ ), whose dynamics is ruled by the phase-correlation kinetic equations (20) and (21). As a remarkable result, the unconstrained thermalization is shown to characterize the equilibrium properties of the waves $(u, v)$ ruled by the NLSE [Eqs. (1) and (2)], such as the amount of energy shared between the pumps and respective sidebands once equilibrium is reached.

In the following, we discuss two different arguments that explain the unexpected zero inverse temperatures [Eq. (30)].

\section{First argument: Helix structure}

The first argument is based on the idea that the constraints imposed by the conservation of Hamiltonian and momentum have no influence on the entropy $\mathcal{S}$. This stems from the simple observation that any surpluses of $\mathcal{H}$ and $\mathcal{P}$ can be stored by "helix" structures that are arbitrarily small in space.

Consider a small spatial subsystem of the whole system that extends from $x_{1}$ to $x_{1}+\Delta x$. Some arbitrary functions are assigned to the field $\left(U_{2}, \alpha_{U}, V_{2}, \alpha_{V}\right)$, where $\alpha_{j}$ simply denote the azimuthal angles in cylindrical coordinates; see Appendix IX B. The angle variables are supposed to increase with constant rates $\partial_{x} \alpha_{U}=a=$ const, $\partial_{x} \alpha_{V}=b=$ const. This contributes

$$
\Delta \mathcal{P}=a \int_{x_{1}}^{x_{1}+\Delta x} U_{2} d x+b \int_{x_{1}}^{x_{1}+\Delta x} V_{2} d x
$$

to the total momentum and

$$
\Delta \mathcal{H}=w a \int_{x_{1}}^{x_{1}+\Delta x} U_{2} d x-w b \int_{x_{1}}^{x_{1}+\Delta x} V_{2} d x
$$

to the Hamiltonian. It is obviously possible to assign any values $a$ and $b$ to this helix structure, so that $\Delta \mathcal{H}$ has an arbitrarily large positive or negative value, while keeping constant the momentum, $\Delta \mathcal{P}=0$. Alternatively, notice that $\Delta \mathcal{P}$ could also have any positive or negative value. We 
stress the idea that this property is fundamentally related to the fact that, because of the convective nature of the interaction $(w \neq 0)$, the sign of the Hamiltonian is undetermined and can be either positive or negative. The helix structure can thus store any positive or negative amount of $\mathcal{H}$ within an arbitrarily small region in space $\Delta x$. At the same time, by virtue of its small size, the formation of such a helix leads only to a negligible change of the entropy $\Delta \mathcal{S}$, so we can associate it with a zero inverse temperature, $\beta=\Delta \mathcal{S} / \Delta \mathcal{H}=0$. On the other hand, the third conserved quantity $\mathcal{M}$ is virtually unaffected, as it is proportional to the small $\Delta x$ but does not contain large values of $a$ or $b$.

In other words, the system can store any positive or negative amount of $\mathcal{H}$ or $\mathcal{P}$ in a helix, but only a negligible amount of $\mathcal{M}$. The helix acts as a source or sink from which the rest of the system can take an arbitrary amount of Hamiltonian and momentum. This allows the rest of the system to achieve the state of maximum disorder without caring for constraints by Hamiltonian or momentum conservation.

Note that this is similar to a first-order phase transition with two coexisting phases. One phase is the narrow helix that absorbs any positive or negative amount of Hamiltonian and momentum, which is possible because these quantities are unbounded and not positive (or negative) definite. The second phase refers to a rapidly fluctuating field that fills almost the entire system and that always contains the right amount of energy and momentum to maximize the entropy, as well as virtually all of $\mathcal{M}$. In this sense, the entropy is not constrained by $\mathcal{H}$ and $\mathcal{P}$, which means that both $\beta$ and $\nu$ are zero.

\section{Second argument: Thermodynamic equilibrium property}

An alternative argument for $\nu=\beta=0$ can be obtained by considering a fundamental thermodynamic equilibrium property, namely, that an isolated system at equilibrium should only exhibit a uniform motion of translation (or rotation) as a whole, while any macroscopic internal motion is not possible at equilibrium [73]. This property means that the maximum of entropy cannot afford to waste energy in macroscopic motion; i.e., turning this energy into the microscopic degrees of freedom creates more entropy. If the system has a nonzero total momentum, this constraint is satisfied with a minimum investment of energy when all subsystems move with the same velocity. A schematic illustration of this thermodynamic equilibrium property is reported in Fig. 6.

We now proceed by following a reasoning similar to that outlined in Ref. [73]. For this purpose, it proves convenient to consider the phase-correlation kinetic model written in terms of the Poincaré-Stokes variables, Eqs. (24) and (25). First, we can divide the system into two macroscopic subsystems corresponding to the two waves $\boldsymbol{U}$ and $\boldsymbol{V}$, with $\mathcal{H}=\mathcal{H}_{U}+\mathcal{H}_{V}+\mathcal{H}_{\text {int }}$; see Eq. (26). It is important to note

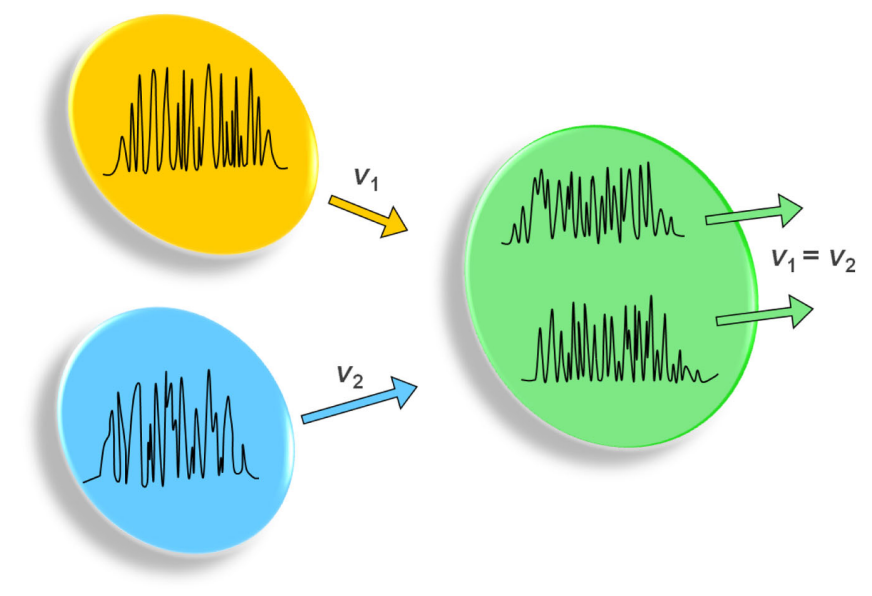

FIG. 6. Property of equilibrium thermodynamics. Schematic illustration of the collision of two incoherent waves with two different velocities. After the collision, the two waves propagate with the same average velocity, so as to prevent a relative internal motion among the two waves: An isolated system at equilibrium can only exhibit a uniform motion of translation as a whole, while any macroscopic internal motion is not possible at equilibrium [73]. This equilibrium thermodynamic property is verified for a system of strongly dispersive (weakly nonlinear) waves, as described by the wave turbulence theory [46]. However, this property is not verified for weakly dispersive (strongly nonlinear) waves ruled by the phase-correlation kinetic equations: A maximum entropy state is achieved for $\nu=\beta=0$; see Eq. (30).

that in the second stage of unconstrained thermalization, the interaction Hamiltonian $\mathcal{H}_{\text {int }}$ is averaged out by the large convection among the two waves $(\varepsilon=1 / w \ll 1$ is the small parameter of the problem), so that the contribution of $\mathcal{H}_{\text {int }}$ results much smaller than the self-interaction contributions, $\mathcal{H}_{\text {int }} \ll \mathcal{H}_{U, V}$ [90]. This important property is confirmed by numerical simulations, as revealed by the good agreement between the numerics and the theoretical equilibrium PDF [Eq. (D2)], in which $\boldsymbol{U}$ and $\boldsymbol{V}$ result uncorrelated with each other.

Because of this weak interaction between $\boldsymbol{U}$ and $\boldsymbol{V}$, near equilibrium the additive entropy can be written in the form $\mathcal{S}=\mathcal{S}_{U}+\mathcal{S}_{V}$. Next, we split each Hamiltonian into the corresponding linear and nonlinear contributions, $\mathcal{H}_{j}=\mathcal{E}_{j}+\mathcal{U}_{j}, \quad j=U, V$ (note that the unconstrained thermalization does not occur in the weakly nonlinear regime, i.e., a priori $\left|\mathcal{E}_{j}\right| \sim\left|\mathcal{U}_{j}\right|$, despite the fact that there is a weak interaction between $\boldsymbol{U}$ and $\boldsymbol{V}$ ). Proceeding as in Ref. [73], the entropy of a subsystem is only a function of its "internal energy," $\mathcal{S}_{j}=\hat{\mathcal{S}}_{j}\left(\mathcal{H}_{j}-\mathcal{E}_{j}\right), \quad j=U, V$. The total entropy as a function of the momentum has a maximum; then by introducing the Lagrange multiplier $\nu$, we look for the maximum of $\mathcal{F}=\sum_{j=U, V} \mathcal{S}_{j}-\nu \mathcal{P}_{j}$. Then $\partial \mathcal{F} / \partial \mathcal{P}_{j}=0$ gives $\nu=-\beta \partial \mathcal{E}_{j} / \partial \mathcal{P}_{j}[73]$.

Let us consider this result in the framework of classical kinetic gas theory. Considering a mixture of two classical gases of masses $M_{j}$, the dispersion relation is quadratic 
$\mathcal{E}_{j}=\mathcal{P}_{j}^{2} /\left(2 M_{j}\right)$, which gives $\mathcal{P}_{j} / M_{j}=-\nu / \beta$ : The two subsystems propagate with the same velocity at equilibrium, $\mathcal{P}_{j} / M_{j}$, which proves the above property of equilibrium thermodynamics [73]. It is important to note that the same equilibrium property holds for a system of weakly nonlinear waves, in which dispersive linear effects dominate nonlinear effects. More precisely, the wave turbulence theory explicitly shows that a set of weakly nonlinear wave packets (with quadratic dispersion relation) relax toward a thermodynamic equilibrium state, in which all wave packets propagate with the same average velocity (see pp. 69-70 in Ref. [46]). In contrast to this weakly nonlinear regime, the macroscopic envelope fluctuations $\left(n_{\varphi_{j}}, m_{\mu}\right)$ evolve in the nonlinear regime of interaction, because the corresponding equations for such correlators are weakly dispersive, as discussed in Sec. IVA. In other terms, the phase-correlation kinetic equations exhibit a purely linear dispersion relation: $\mathcal{E}_{U}=w \mathcal{P}_{U}, \mathcal{E}_{V}=-w \mathcal{P}_{V}$; see Eq. (26). In this way, $\partial \mathcal{F} / \partial \mathcal{P}_{U}=0$ gives $\nu=-\beta w$, whereas $\partial \mathcal{F} / \partial \mathcal{P}_{V}=0$ gives $\nu=+\beta w$, which thus leads to the conclusion $\nu=\beta=0$. As a consequence, weakly dispersive systems like Eqs. [(20) and (21)] [or Eqs. (24) and (25)] cannot satisfy the above equilibrium property, so that the maximum entropy state is achieved with $\nu=\beta=0$.

\section{Equilibrium PDFs for the normal and anomalous correlators}

According to the previous discussion on zero inverse temperatures, $\beta=\nu=0$, the general form of the PDF Eq. (29) reduces to $\rho \sim \exp (-\gamma \mathcal{M})$. Starting from this reduced density, we compute the marginal PDFs of the normal and anomalous phase correlators. Specifically, the PDFs for $n_{\varphi_{p}}^{0}(\varphi=u, v)$ are exponential truncated on $\left[0, N_{\varphi}\right]$ :

$p\left(n_{\varphi_{p}}^{0}\right)=\frac{\gamma \exp \left[s \gamma\left(N_{\varphi} / 2-n_{\varphi_{p}}^{0}\right)\right]}{2 \sinh \left(\gamma N_{\varphi} / 2\right)} \Pi\left(1-2 n_{\varphi_{p}}^{0} / N_{\varphi}\right)$,

where $s=+1$ for $\varphi=u(s=-1$ for $\varphi=v)$ and $\Pi(x)=1$ if $x \in[-1,1]$ and 0 otherwise. For $N_{u}=N_{v}$, then $\gamma=0$, and the PDFs $p\left(n_{\varphi_{p}}^{0}\right)$ become uniform over $\left[0, N_{\varphi}\right]$, which contrasts with Gaussian statistics. On the other hand, the marginal PDF for the anomalous phase correlator reads

$p\left(\left|m_{\mu}^{0}\right|\right)=\frac{\gamma\left|m_{\mu}^{0}\right| \cosh \left(\gamma \sqrt{N_{\varphi}^{2} / 4-\left|m_{\mu}^{0}\right|^{2}}\right)}{\sinh \left(\gamma N_{\varphi} / 2\right) \sqrt{N_{\varphi}^{2} / 4-\left|m_{\mu}^{0}\right|^{2}}} \Pi\left(1-\frac{4\left|m_{\mu}^{0}\right|}{N_{\varphi}}\right)$,

for $(\mu=u v, \varphi=u)$ or $(\mu=v u, \varphi=v)$.

From the PDFs [Eqs. (33) and (34)], we obtain the averages of the normal and anomalous correlators:

$$
\left\langle n_{\varphi_{p}}^{0}\right\rangle_{\mathrm{eq}}=\frac{N_{\varphi}}{2}+\frac{s}{\gamma}-\frac{s N_{\varphi}}{2 \tanh \left(\gamma N_{\varphi} / 2\right)},
$$

while

$$
\left\langle\left|m_{\mu}^{0}\right|\right\rangle_{\mathrm{eq}}=\frac{\pi N_{\varphi} I_{1}\left(\gamma N_{\varphi} / 2\right)}{4 \sinh \left(\gamma N_{\varphi} / 2\right)},
$$

and $\left\langle\operatorname{Re}\left(m_{\mu}^{0}\right)\right\rangle_{\mathrm{eq}}=\left\langle\operatorname{Im}\left(m_{\mu}^{0}\right)\right\rangle_{\mathrm{eq}}=0$, where $I_{1}(x)$ is the firstorder modified Bessel function of first kind. The expressions of the PDFs of the correlators and the corresponding averages are found in good agreement with the simulations of the phase-correlation kinetic equations (20) and (21), as illustrated in Fig. 7.

We remark that the PDF distributions [Eqs. (33) and (34)] are of a different nature than Gaussian statistics, which corroborates the fact that the dynamics ruled by the phase-correlation kinetic equations cannot be described by a weakly nonlinear approach, such as the wave turbulence theory. Let us recall that the above PDF distributions describe the averaged envelopes of the macroscopic nonhomogeneous spatial fluctuations of the waves $u(x, t)$ and $v(x, t)$, while the underlying microscopic fluctuations (on the small scale $\lambda_{c}$ ) ruled by NLSE [Eqs. (1) and (2)] still exhibit Gaussian statistics. More precisely, the phase-correlation kinetic equations (20) and (21) are derived under the assumption that the correlators $\left[n_{\varphi_{j}}^{0}(x, t), m_{\mu}^{0}(x, t)\right]$ evolve with a macroscopic correlation length scale, of the order $\ell_{c} \sim w \gg 1$. During their
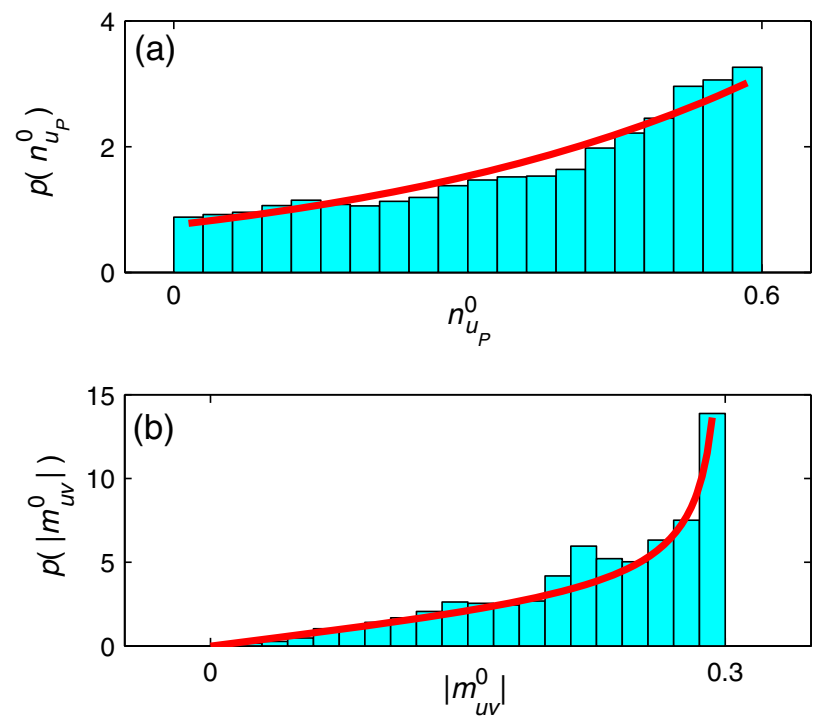

FIG. 7. Unconstrained thermalization: PDFs of the correlators. Equilibrium PDFs of the normal correlator $n_{u_{p}}^{0}$ (a) and anomalous phase correlator $\left|m_{u v}^{0}\right|$ (b), obtained from simulations of the phase correlation kinetic equations (20) and (21). A good agreement is obtained with the theory; see the equilibrium PDFs [Eqs. (33) and (34)] in red lines. The equilibrium state is not constrained by Hamiltonian and momentum conservation $\left(N_{u}=0.6, N_{v}=0.4\right.$, $\kappa=2 / 3, w=54)$. 
relaxation to equilibrium, the typical correlation lengths of $\left[n_{\varphi_{j}}^{0}(x, t), m_{\mu}^{0}(x, t)\right]$ get smaller and smaller, so that there exists some time beyond which such correlation lengths are much smaller than $w$, which thus invalidates the assumption made to derive the phase-correlation kinetic equations. In computing the PDFs of the correlators $\left(n_{\varphi_{j}}, m_{\mu}\right)$ numerically, the simulations are stopped before the envelope kinetic equations (20) and (21) break down, i.e., for spectral widths not much larger than $2 \pi / w$ (typically for $t \lesssim 200$ ). The PDFs we compute numerically then approach the corresponding PDFs predicted by our equilibrium theory within some large, yet finite, time.

A remarkable result revealed by the unconstrained thermalization of the correlators $\left(n_{\varphi_{j}}^{0}, m_{\mu}^{0}\right)$ is that it provides an analytical expression of the energy shared between the pumps and respective sidebands at equilibrium. This is expressed by Eq. (35), which gives the amount of energy in the pump components, $\left\langle n_{\varphi_{p}}^{0}\right\rangle_{\mathrm{eq}}=\left\langle\left|\varphi_{p}(x, t)\right|^{2}\right\rangle_{\mathrm{eq}}$, with $\varphi=u, v$, while the equilibria for the sidebands $\left\langle n_{\varphi_{s}}^{0}\right\rangle_{\mathrm{eq}}$ are deduced from energy conservation, $N_{\varphi}=$ const. These analytical expressions of the pump and sideband energies are found in quantitative agreement with NLSE simulations without using adjustable parameters, as illustrated in Fig. 5.

\section{DISCUSSION}

In summary, we show that large convection among weakly interacting random waves is responsible for a phenomenon of incoherent recurrence that can occur far from integrability and that originates in the spontaneous emergence of strongly correlated fluctuations. We explain this phenomenon by developing a nonequilibrium kinetic formulation accounting for spatial nonhomogeneous phase correlations. The theory reveals that the incoherent recurrence is due to a novel form of modulational instability that occurs solely for the phase correlations $m_{\mu}$, so that the growths of the usual modulational unstable sidebands are delayed by a significant time. In contrast to conventional incoherent modulational instabilities, we identify a regime in which Landau damping is completely suppressed, which unexpectedly eliminates the modulational instability threshold. Owing to this remarkable fact, the incoherent recurrence can take place for strongly incoherent waves in the weakly nonlinear regime, which thus leads to a violation of the $H$ theorem of entropy growth.

After a few incoherent recurrences, the system enters a secondary turbulent regime for the correlators $\left(n_{\varphi_{j}}, m_{\mu}\right)$, which is characterized by an irreversible evolution to equilibrium. This thermalization process cannot be described by a weakly nonlinear treatment, so we resort to a Gibbsian statistical mechanics approach on the correlators. Unexpectedly, the analysis reveals the existence of a novel scenario of irreversible thermalization, which is not constrained by energy (Hamiltonian) and momentum conservation; i.e., the corresponding inverse temperatures are zero, $\beta=\nu=0$. We give two physical arguments without a rigorous mathematical proof that explain the nature of the unconstrained thermalization process. The first one is based on the idea that the presence of narrow helix-shaped coherent structures can store any amount of the Hamiltonian and momentum, so that the constraints imposed by these conserved quantities have no influence on the entropy. The second argument is based on thermodynamics grounds and reveals that the unconstrained thermalization does not verify an equilibrium property, namely, that an isolated system at equilibrium should exhibit a uniform motion of translation as a whole. Both arguments rely on the fact that the dynamics is dominated by the convection among the waves, which introduces an undetermined sign of the energy (the Hamiltonian is not positive or negative definite). Our numerical simulations are in good agreement with the equilibrium PDFs predicted by the unconstrained thermalization process. In this respect, it is interesting to note that the equilibrium PDFs of the macroscopic fluctuations of the waves exhibit strong deviations from Gaussianity, which shows that a bunch of random waves with large intensity ("incoherent rogue wave") can even be more probable than bunches of small intensities (see Fig. 7).

\section{A. Generality of the process of unconstrained thermalization}

Her, we emphasize that the unconstrained thermalization does not constitute a specific property of the phasecorrelation kinetic equations derived in our paper, but rather a general property for weakly dispersive wave systems whose dynamics is dominated by convection. We illustrate such a generality by considering two important examples.

We first consider the evolution of a nonlinear wave in a periodic potential, a problem encountered in a variety of physical disciplines, such as optics, condensed matter physics, or Bose-Einstein condensates [91-94], in which the behavior of atoms mimics those of electrons in crystals or photons in optical gratings. Because of Bragg reflections around a forbidden frequency band gap, these systems are generally characterized by a counterpropagating wave interaction, so that the dynamics is dominated by convection; see Appendix E. The corresponding model has been widely studied in relation with the generalized massive Thirring model [91-94]. Note that this model is also relevant to the description of ocean waves in deep water for a periodic bottom [95].

Another remarkable example of weakly dispersive wave system is provided by the resonant three-wave interaction, which is known to occur in any weakly nonlinear medium whose lowest-order nonlinearity is quadratic in terms of the wave amplitudes. For this reason, the three-wave interaction is encountered in such diverse fields as plasma physics, hydrodynamics, acoustics, and nonlinear optics [96]. 
Both the Thirring model and the three-wave interaction model constitute weakly dispersive systems with purely linear dispersion relations. Accordingly, these systems exhibit a Hamiltonian structure analogous to that of the phase-correlation kinetic equations; see Appendix E. Hence, following the analysis developed in Sec. IV C, both models exhibit an irreversible process of thermalization toward an equilibrium which is not constrained by energy (Hamiltonian) and momentum conservation. We finally note that, as discussed above through the phase-correlation kinetic equations, the equilibrium state itself contains arbitrarily short fluctuations, in a way similar to the well-known ultraviolet catastrophe inherent to the ensemble of classical waves [39]. In this respect, the equilibrium is not, strictly speaking, a physical state. Its physical importance is its role as a statistical attractor that governs the thermalization process at physically relevant scales.

\section{B. Degenerate resonances}

It is interesting to note that for $\eta=1$, the dispersion relations of the system [Eqs. (1) and (2)] exhibit degenerate resonances, a property of fundamental importance to find additional integral invariants of kinetic equations [97], in relation to the general problem of integrability $[15,98]$. It turns out that the wave turbulence kinetic equations associated with Eqs. (1) and (2) admit "local" invariants for $\eta=1$ [99]. Contrary to usual integral invariants which lead to a generalized Rayleigh-Jeans distribution [97], local invariants are responsible for an anomalous process of thermalization toward an equilibrium state of a different nature than the Rayleigh-Jeans spectrum [99]. However, we stress the fact that, irrespective of the local or integral nature of the invariants, the wave turbulence theory still predicts a monotonic process of entropy production. Therefore, the wave turbulence theory cannot describe the incoherent recurrences reported here, despite the fact that they occur in the weakly nonlinear regime. We note the interesting aspect that fast oscillatory energy transfers among modes can be described through quasiresonant (or nonresonant) interactions in the framework of a generalized version of the wave turbulence kinetic equation (see Refs. [71,100,101] and Chap. 7 in Ref. [41]). However such a generalized kinetic approach does not account for the existence of phase correlations, so that it is inherently unable to describe the incoherent recurrences we report here.

We also note that the process of "anomalous thermalization" reported in Ref. [99] is of a fundamentally different nature than the unconstrained thermalization reported in the present work. Firstly, as discussed above, the anomalous thermalization results from the conservation of a local invariant in frequency space, which implies, in particular, the conservation of the energy and momentum [99]. Then in contrast to the unconstrained thermalization process, the anomalous thermalization is constrained by the conservation of energy and momentum. Secondly, the anomalous thermalization process is entirely described by the standard wave turbulence kinetic equations in the weakly nonlinear regime [99]. This is in contrast to the unconstrained thermalization that occurs for weakly dispersive wave systems that evolve in the strongly nonlinear regime, as revealed by the equilibrium PDFs, which are of a different nature than Gaussian statistics (see Sec. IV D).

\section{Experimental implementations}

Spatiotemporal recurrence phenomena have been widely studied with purely coherent waves in various different recent experiments in optical fibers [26,27,34] and water tanks $[29,30,34,35]$. The present work should stimulate a novel class of experiments aimed at observing spatiotemporal recurrences with incoherent waves.

The phenomenon of incoherent recurrence reported here should be observable in coupled Bose-Einstein condensates [78]. One may simply consider a binary mixture of the same atomic species (same masses) with different internal degrees of freedom, so that $\eta=1$ in Eqs. (1) and (2), while the amount of convection among the waves can be controlled by well-known techniques [102]. We remark that this type of experiment is relevant to the emergent key area of quantum turbulence in Bose-Einstein condensates [103]. Hydrodynamic experiments can also be envisaged in water tanks, by considering the interaction of deep water waves propagating in different directions (note that $\eta=1$ in the NLSE model [76]).

We also consider with care the feasibility of an experiment in an optical fiber system. In this respect, the NLSE [Eqs. (1) and (2)] are known to accurately describe the propagation of orthogonal polarization components $(u, v)$ in highly birefringent optical fibers. In this setting, the cross-phase modulation coefficient is set to $\kappa=2 / 3$ (which corresponds to the value used in the simulations) and the ratio between the dispersion coefficients is usually approximated to $\eta \simeq 1$, while the convection among the polarization components $u$ and $v$ is related to the amount of fiber birefringence [74]. Our analysis reveals that the numerical simulations reported in this paper refer to an experiment that can be implemented with currently available fiber technology, so that we can reasonably expect in the near future the observation of the phenomenon of incoherent recurrence.

\section{Toward incoherent breathers}

The spatiotemporal character of the kinetic formulation we develop in this work paves the way to the study of novel forms of global incoherent collective behaviors in turbulent systems. In the following, we discuss the important example of breathers [27-35,104-109]. In this respect, we note that, although breather structures have been shown to emerge from a turbulent state of the system $[104,105,108,109]$, the breather itself has always been considered as being inherently a coherent localized entity. 
On the other hand, we show in this work that, by creating strong phase correlations, convection introduces a largescale behavior of the incoherent wave $\ell_{c} \sim w$ [see Eq. (17)], whose macroscopic envelope evolution is described in detail by the phase-correlation kinetic theory. Now, one can simply remark that a coherent breather solution of the phasecorrelation kinetic equations (24) and (25) corresponds to an incoherent breather state by referring back to the original NLSE random waves. In other words, the incoherent waves $(u, v)$ ruled by the NLSE system exhibit a microscopic incoherent random structure, and a macroscopic envelope structure, $\left(\boldsymbol{U}_{b r}, \boldsymbol{V}_{b r}\right)$, which corresponds to a coherent breather solution of the kinetic equations (24) and (25). Then in contrast to widely studied coherent breathers, our formulation predicts the existence of a large-scale incoherent breather structure, in which it is the incoherent wave as a whole that exhibits spatiotemporal recurrences.

\section{ACKNOWLEDGMENTS}

J.F., M.G., and A.P. acknowledge funding from the European Research Council under the European Community's Seventh Framework Programme (FP7/ 2007-2013 Grant Agreement No. 306633, PETAL project). M. G., J. F., F. A., and A. P. acknowledge support from the French National Research Agency (ANR-12-BS04-0011 OPTIROC) and the Labex ACTION (ANR-11-LABX-0101) program. B. R. acknowledges a grant from the Simons Foundation (No. 430192). D. S. acknowledges support from the Technische Universitat München Institute for Advanced Study, funded by the German Excellence Initiative and the European Union Seventh Framework Programme under Grant Agreement No. 291763. M. G. acknowledges support from the Marie Sklodowska-Curie Fellowship IF project AMUSIC-702702. A. P. acknowledges P. Suret and S. Randoux for stimulating discussions on their preliminary experimental results during the early stages of the work. A. P. is also grateful to M. Onorato for pointing out Ref. [10] and the discussion relative to the proximity to integrability.

\section{APPENDIX A: INAPPLICABILITY OF THE WAVE TURBULENCE THEORY}

The standard mathematical tool to study the dynamics of weakly nonlinear random waves is based on the wave turbulence theory [36,38-40]. This kinetic approach relies on a natural asymptotic closure of the moments equations, which is induced by the dispersive properties of the waves. It leads to a kinetic description of the wave interaction that is formally based on irreversible kinetic equations, a feature which is expressed by an $H$ theorem of entropy growth. The wave turbulence theory is essentially based on the following assumptions: (i) the waves evolve in a regime of weak nonlinear interaction $|\tilde{U} / \tilde{E}| \ll 1$, (ii) the waves exhibit fluctuations that are statistically homogeneous in space, (iii) there are no correlations among the wave components $(u, v)$. In this way, the theory derives a set of irreversible kinetic equations that govern the evolutions of the averaged spectra of the fields $\tilde{n}_{u}(k, t)$ and $\quad \tilde{n}_{v}(k, t): \quad\left\langle\tilde{u}\left(k_{1}, t\right) \tilde{u}^{*}\left(k_{2}, t\right)\right\rangle=\tilde{n}_{u}\left(k_{1}, t\right) \delta\left(k_{1}-k_{2}\right)$, $\left\langle\tilde{v}\left(k_{1}, t\right) \tilde{v}^{*}\left(k_{2}, t\right)\right\rangle=\tilde{n}_{v}\left(k_{1}, t\right) \delta\left(k_{1}-k_{2}\right)$, with $\tilde{u}(k, t)=$ $(1 / \sqrt{2 \pi}) \int u(x, t) \exp (-i k x) d x, \tilde{v}(k, t)=(1 / \sqrt{2 \pi}) \int v(x, t) \times$ $\exp (-i k x) d x$. Following the wave turbulence procedure, one obtains the following set of coupled kinetic equations:

$$
\begin{aligned}
& \partial_{t} \tilde{n}_{u}(k, t)=\frac{\kappa^{2}}{2 \pi} \iiint d k_{1} d k_{2} d k_{3} \mathbf{R}_{u v} W_{u v}, \\
& \partial_{t} \tilde{n}_{v}(k, t)=\frac{\kappa^{2}}{2 \pi} \iiint d k_{1} d k_{2} d k_{3} \mathbf{R}_{v u} W_{v u},
\end{aligned}
$$

where $\mathbf{R}_{u v}=\tilde{n}_{u}\left(k_{1}\right) \tilde{n}_{v}\left(k_{2}\right) \tilde{n}_{v}\left(k_{3}\right) \tilde{n}_{u}(k)\left[\tilde{n}_{u}^{-1}(k)+\tilde{n}_{v}^{-1}\left(k_{3}\right)-\right.$ $\left.\tilde{n}_{v}^{-1}\left(k_{2}\right)-\tilde{n}_{u}^{-1}\left(k_{1}\right)\right], \quad W_{u v}=\delta\left(\omega_{u}\left(k_{1}\right)+\omega_{v}\left(k_{2}\right)-\omega_{v}\left(k_{3}\right)-\right.$ $\left.\omega_{u}(k)\right) \delta\left(k_{1}+k_{2}-k_{3}-k\right)$, while $\mathbf{R}_{v u}$ and $W_{v u}$ are deduced with the substitutions $u \leftrightarrow v$. The dispersion relations read $\omega_{u}(k)=k^{2}+w k, \omega_{v}(k)=\eta k^{2}-w k$. The resonant conditions of energy and momentum conservation are expressed by the Dirac $\delta$ functions. Note that the self-interaction term in the NLSE [Eqs. (1) and (2)] does not contribute to the kinetic equations, because the conservations of energy and momentum are trivially satisfied in one dimension. Equations (A1) and (A2) conserve the energies (number of particles) of each component, $N_{\varphi}=\int \tilde{n}_{\varphi}(k, t) d k$, the total momentum, $\tilde{P}=\sum_{\varphi} \tilde{P}_{\varphi}$, $\tilde{P}_{\varphi}=\int k \tilde{n}_{\varphi}(k, t) d k$, and the linear contribution to the Hamiltonian, $\tilde{E}=\sum_{\varphi} \tilde{E}_{\varphi}, \quad \tilde{E}_{\varphi}=\int \omega_{\varphi}(k) \tilde{n}_{\varphi}(k, t) d k$ $(\varphi=u, v)$. The irreversible character of Eqs. (A1) and (A2) is expressed by an $H$ theorem of entropy growth, $d \tilde{\mathcal{S}} / d t \geq 0$, where $\tilde{\mathcal{S}}=\sum_{\varphi} \tilde{\mathcal{S}}_{\varphi}$, and $\tilde{\mathcal{S}}_{\varphi}(t)=\int \log \left[\tilde{n}_{\varphi}(k, t)\right] d k$ is the nonequilibrium entropy of the $\varphi$ th component $(\varphi=u, v)$. The thermodynamic equilibrium spectra $\tilde{n}_{\varphi}^{\mathrm{RJ}}(k)$ realizing the maximum of entropy $\tilde{\mathcal{S}}\left[\tilde{n}_{u}, \tilde{n}_{v}\right]$, given the constraints of conservation of $\tilde{E}, \tilde{P}$, and $\tilde{N}_{\varphi}$, refer to the well-known Rayleigh-Jeans distribution,

$$
\tilde{n}_{\varphi}^{\mathrm{RJ}}(k)=\frac{\tilde{T}}{\omega_{\varphi}(k)+\tilde{\nu} k-\tilde{\mu}_{\varphi}}, \quad \varphi=u, v,
$$

where $\tilde{T}$ (temperature), $\tilde{\mu}_{u, v}$ (chemical potentials), and $\tilde{\nu}$ are the Lagrangian multipliers associated to $\tilde{E}, \tilde{N}_{\varphi}, \tilde{P}$, respectively. Energy equipartition among the modes takes place in the tails (large $k$ ) of the Rayleigh-Jeans distribution, where $\omega_{\varphi}(k) \tilde{n}_{\varphi}^{\mathrm{RJ}}(k) \simeq \tilde{T}$. Regardless of the amount of convection in the system, i.e., irrespective of $w$, the kinetic equations (A1) and (A2) then describe an irreversible thermalization to the Rayleigh-Jeans spectrum, so that the wave turbulence theory is unable to describe the incoherent recurrences. 


\section{APPENDIX B: DERIVATION OF THE PHASE-CORRELATION KINETIC EQUATIONS [EQS. (11) AND (12)]}

\section{General form of the kinetic equations}

The starting point consists of splitting each wave, $u(x, t)$ and $v(x, t)$, into a pump component and the corresponding sideband modulational instability spectral component:

$$
\begin{aligned}
& u(x, t)=\tilde{u}_{p}(x-w t, t)+\tilde{u}_{s}(x+w t, t) \exp (-i w x), \\
& v(x, t)=\tilde{v}_{p}(x+w t, t)+\tilde{v}_{s}(x-w t, t) \exp (+i w x),
\end{aligned}
$$

with the assumption that $w \gg \sigma$, where $\sigma$ is the typical spectral bandwidth of the waves $\tilde{u}_{j}$ and $\tilde{v}_{j}(j=p, s)$. The coherence length of a field, $\lambda_{c} \sim 2 \pi / \sigma$, is typically smaller than one within our normalization. The shifts $\pm w \partial_{x}$ in Eqs. (B1) and (B2) are chosen so as to cancel the transport terms in the four-wave model equations. Substituting these Ansätze into the NLSE, and keeping the resonant terms, we obtain:

$$
i \partial_{t} \tilde{u}_{p}=\mathcal{F}_{p}\left[\tilde{u}_{j}, \tilde{v}_{j}\right]+\kappa \tilde{v}_{s}(x)\left(\tilde{u}_{s} \tilde{v}_{p}^{*}\right)(x+2 w t)-\partial_{x x} \tilde{u}_{p},
$$

$i \partial_{t} \tilde{u}_{s}=\mathcal{F}_{s}\left[\tilde{u}_{j}, \tilde{v}_{j}\right]+\kappa \tilde{v}_{p}(x)\left(\tilde{u}_{p} \tilde{v}_{s}^{*}\right)(x-2 w t)-\partial_{x x} \tilde{u}_{s}$,

with $(j=p, s)$, and where the functionals read $\mathcal{F}_{p}\left[\tilde{u}_{j}, \tilde{v}_{j}\right]=$ $\left[\left|\tilde{u}_{p}\right|^{2}+2\left(\left|\tilde{u}_{s}\right|^{2}\right)(x+2 w t)+\kappa\left(\left|\tilde{v}_{p}\right|^{2}\right)(x+2 w t)+\kappa\left|\tilde{v}_{s}\right|^{2}\right] \tilde{u}_{p}$, and $\mathcal{F}_{s}\left[\tilde{u}_{j}, \tilde{v}_{j}\right]=\left[\left|\tilde{u}_{s}\right|^{2}+2\left(\left|\tilde{u}_{p}\right|^{2}\right)(x-2 w t)+\kappa\left|\tilde{v}_{p}\right|^{2}+\right.$ $\left.\kappa\left(\left|\tilde{v}_{s}\right|^{2}\right)(x-2 w t)\right] \tilde{u}_{s}$. The equations for $\left(\tilde{v}_{p}, \tilde{v}_{s}\right)$ can be derived from those for $\left(\tilde{u}_{p}, \tilde{u}_{s}\right)$ with the substitutions $\tilde{u}_{j} \leftrightarrow \tilde{v}_{j}(j=p, s), w \rightarrow-w, \partial_{x x} \tilde{u}_{j} \rightarrow \eta \partial_{x x} \tilde{v}_{j}(j=p, s)$. Note that, contrary to the usual four-wave interaction equations, we retain here second-order dispersion effects [last terms in Eqs. (B3) and (B4)], which appear to play a fundamental role in the emergence of phase correlations among the random waves. Specifically, the phasecorrelation modulational instability reveals that the characteristic length scale of nonhomogeneous statistics of the random waves is determined by the amount of convection in the system, $\ell_{c} \sim w$ [see Eq. (17)]. The existence of incoherent recurrences requires the presence of a large convection, $w \gg 1$. Then the microscopic scale fluctuations of the random waves that are characterized by the coherence length, $\lambda_{c} \sim 1$, are much smaller than the macroscopic scale variations of the nonhomogeneous statistics, i.e., $\lambda_{c} \ll w$. In this way, large convection is responsible for an effective averaging of the microscopic scale fluctuations.

We derive an ensemble of closed equations for the evolutions of the correlation functions that describe the dynamics of the macroscopic scale fluctuations of the random waves. Under the assumption that the interaction takes place in the weakly nonlinear regime, we follow the general procedure of the wave turbulence theory to achieve a closure of the infinite hierarchy of moments equations. However, at variance with the standard wave turbulence theory, here, we take into account the existence of phasecorrelation effects among the two pairs of copropagating random wave components, as well as the nonhomogeneous statistics of the macroscopic spatial fluctuations. We denote $\quad u_{p}(x, t)=\tilde{u}_{p}(x-w t, t), u_{s}(x, t)=\tilde{u}_{s}(x+w t, t)$, $v_{p}(x, t)=\tilde{v}_{p}(x+w t, t), v_{s}(x, t)=\tilde{v}_{s}(x-w t, t)$. The correlation functions are defined as follows: $B_{\varphi_{j}}(x, \xi, t)=$ $\left\langle u_{j}(x+\xi / 2, t) u_{j}^{*}(x-\xi / 2, t)\right\rangle$, for $\varphi=u, v(j=p, s)$, and $\Gamma_{u v}(x, \xi, t)=\left\langle u_{p}(x+\xi / 2, t) v_{s}^{*}(x-\xi / 2, t)\right\rangle, \Gamma_{v u}(x, \xi, t)=$ $\left\langle u_{s}(x+\xi / 2, t) v_{p}^{*}(x-\xi / 2, t)\right\rangle$. Recalling that the large length scale of macroscopic fluctuations associated to the nonhomogeneous statistics is $\ell_{c} \sim w \gg 1$, we consider a multiscale expansion with the small parameter $\varepsilon=1 / w$ :

$$
\begin{aligned}
B_{\varphi_{j}}(x, \xi, t) & =B_{\varphi_{j}}^{(0)}(\varepsilon x, \xi, t), \quad \varphi=u \quad \text { or } \quad \varphi=v(j=p, s), \\
\Gamma_{\varphi}(x, \xi, t) & =\Gamma_{\varphi}^{(0)}(\varepsilon x, \xi, t), \quad \varphi=u v \quad \text { or } \quad \varphi=v u .
\end{aligned}
$$

In this way, we have $w \partial_{x} B_{\varphi_{j}}(x, \xi, t)=\partial_{X} B_{\varphi_{j}}^{(0)}(X, \xi, t)$, with $X=\varepsilon x(\varphi=u, v, j=p, s)$. We also have $B_{\varphi_{j}}(x \pm \xi / 2,0, t)-$ $B_{\varphi_{j}}(x \mp \xi / 2,0, t) \simeq \pm \varepsilon \xi \partial_{X} B_{\varphi_{j}}^{(0)}(X, 0, t)$, so that these terms result to be negligible with respect to terms of the form $w \partial_{x} B_{\varphi_{j}}(x, \xi, t)$. In this regime, second-order dispersion effects (second-order spatial derivatives in the NLSE) are negligible for the autocorrelation functions, $\partial_{x \xi}^{2} B_{\varphi_{j}}(x, \xi)=$ $\varepsilon \partial_{X \xi}^{2} B_{\varphi_{j}}^{(0)}(X, \xi, t)$, while dispersion effects do affect the evolutions of the phase-correlation functions with terms of the form $\partial_{\xi}^{2} \Gamma_{u v}^{(0)}(X, \xi, t)$. Collecting terms of the order $\varepsilon^{0}$, we obtain the following system of closed equations governing the evolutions of the correlation functions:

$$
\begin{aligned}
i\left(\partial_{t}+w \partial_{x}\right) B_{u_{p}}(x, \xi) & =\mathcal{B}_{u_{p}}\left[\Gamma_{\mu}\right], \\
i\left(\partial_{t}-w \partial_{x}\right) B_{u_{s}}(x, \xi) & =\mathcal{B}_{u_{s}}\left[\Gamma_{\mu}\right]
\end{aligned}
$$

$i\left(\partial_{t}+w \partial_{x}\right) \Gamma_{u v}(x, \xi)=\mathcal{B}_{u v}\left[B_{\varphi_{j}}, \Gamma_{\mu}\right]-\delta \partial_{\xi}^{2} \Gamma_{u v}(x, \xi)$,

where $\delta=1-\eta$ and the functionals read $\mathcal{B}_{u_{p}}\left[\Gamma_{\mu}\right]=$ $-\kappa \Gamma_{u v}(x, \xi)\left[\Gamma_{u v}^{*}(x, 0)+\Gamma_{v u}^{*}(x, 0)\right]+\kappa \Gamma_{u v}^{*}(x,-\xi)\left[\Gamma_{u v}(x, 0)+\right.$ $\left.\Gamma_{v u}(x, 0)\right], \quad \mathcal{B}_{u_{s}}\left[\Gamma_{\mu}\right]=-\kappa \Gamma_{v u}(x, \xi)\left[\Gamma_{u v}^{*}(x, 0)+\Gamma_{v u}^{*}(x, 0)\right]+$ $\kappa \Gamma_{v u}^{*}(x,-\xi)\left[\Gamma_{u v}(x, 0)+\Gamma_{v u}(x, 0)\right], \quad$ and $\mathcal{B}_{u v}\left[B_{\varphi_{j}}, \Gamma_{\mu}\right]=$ $\Gamma_{u v}(x, \xi)\left\{\kappa\left[B_{v_{p}}(x, 0)-B_{u_{p}}(x, 0)+B_{v_{s}}(x, 0)-B_{u_{s}}(x, 0)\right]+\right.$ $\left.2\left[B_{u_{p}}(x, 0)-B_{v_{s}}(x, 0)+B_{u_{s}}(x, 0)-B_{v_{p}}(x, 0)\right]\right\}+\kappa\left[B_{v_{s}}(x, \xi)-\right.$ $\left.B_{u_{p}}(x, \xi)\right]\left[\Gamma_{u v}(x, 0)+\Gamma_{v u}(x, 0)\right]$. The corresponding equations for $B_{v_{j}}(x, \xi, t)(j=p, s)$ and $\Gamma_{v u}(x, \xi, t)$ can be deduced with the substitutions: $\tilde{u}_{j} \leftrightarrow \tilde{v}_{j}(j=p, s)$, $w \rightarrow-w, \Gamma_{u v}(x, \xi, t) \rightarrow \Gamma_{v u}^{*}(x,-\xi, t), \delta \rightarrow-\delta$. 


\section{Invariant correlation functions}

Equations (B5)-(B7) reveal the existence of conserved correlation functions: $\left(\partial_{t}+w \partial_{x}\right)\left[B_{u_{p}}(x, \xi, t)+B_{v_{s}}(x, \xi, t)\right]=0$ and $\left(\partial_{t}-w \partial_{x}\right)\left[B_{v_{p}}(x, \xi, t)+B_{u_{s}}(x, \xi, t)\right]=0$. Let us analyze these invariant correlation functions in the problem under consideration here, in which only the two incoherent pump waves are initially injected in the system; i.e., $\left|\varphi_{p}(x, t=0)\right| \gg\left|\varphi_{s}(x, t=0)\right|$, with $\varphi=u, v$. Moreover, the incoherent pump waves exhibit fluctuations that are statistically homogeneous in space, so that we have two conserved functions, $Q_{u}(\xi)$ and $Q_{v}(\xi)$, which are determined by the initial condition

$$
\begin{aligned}
& Q_{u}(\xi)=B_{u_{p}}(\xi, t=0)=B_{u_{p}}(x, \xi, t)+B_{v_{s}}(x, \xi, t), \\
& Q_{v}(\xi)=B_{v_{p}}(\xi, t=0)=B_{v_{p}}(x, \xi, t)+B_{u_{s}}(x, \xi, t) .
\end{aligned}
$$

Note that these invariants do not depend on the spatial variable $x$, because of the assumption of homogeneous statistics of the initial pump waves. Owing to the invariants [Eqs. (B8) and (B9)], the general equations for the correlation functions Eqs. (B5)-(B7) reduce to a set of four closed equations. These can be reformulated by considering the evolutions of the local spectra of the fields by taking the Wigner-like transform, as defined through Eqs. (9) and (10). In this way, we obtain the phase-correlation kinetic equations (11) and (12) for the evolutions of $n_{u_{p}}(x, k, t)$ and $m_{v u}(x, k, t)$, where $N_{\varphi}=$ $Q_{\varphi}(\xi=0)$ (with $\varphi=u, v$ ) denote the conserved "energies" of the waves $u$ and $v$. Note that the corresponding equations for $n_{v_{p}}(x, k, t)$ and $m_{u v}(x, k, t)$ can be deduced from Eqs. (11) and (12) through the substitution $n_{u_{p}} \leftrightarrow n_{v_{p}}, w \rightarrow-w$, $m_{v u}(x, \xi, t) \leftrightarrow m_{u v}^{*}(x, \xi, t),(1-\eta) \rightarrow-(1-\eta)$.

\section{Formal reversibility}

Note that, contrary to the usual wave turbulence formalism discussed in Appendix A [36,39,40], the system Eqs. (11) and (12) is reversible in time; i.e., the equations are invariant under the transformation

$t \rightarrow-t, \quad w \rightarrow-w, \quad k \rightarrow-k, \quad n_{\varphi} \rightarrow n_{\varphi}, \quad m_{\mu} \rightarrow m_{\mu}^{*}$.

This transformation is inherited from the well-known time invariance of the NLSE [Eqs. (1) and (2)]: $t \rightarrow-t$, $w \rightarrow-w, u \rightarrow u^{*}, v \rightarrow v^{*}$.

\section{APPENDIX C: HAMILTONIAN STRUCTURE OF THE PHASE-CORRELATION KINETIC EQUATIONS}

\section{Spherical coordinates}

The Hamiltonian of Eqs. (20) and (21) can be unveiled by using the Poincaré-Stokes representation reported in Eqs. (24) and (25). We consider the Poisson bracket:

$$
\{F, G\}=\sum_{i j k} \int d x^{\prime} \delta\left(x-x^{\prime}\right) \varepsilon_{i j k} \mathcal{Q}[F, G],
$$

where $\mathcal{Q}[F, G]=U_{i}\left[\delta F /\left(\delta U_{j}\right)\right]\left[\delta G /\left(\delta U_{k}\right)\right]+V_{i}\left[\delta F /\left(\delta V_{j}\right)\right]$ $\left[\delta G /\left(\delta V_{k}\right)\right]$, and $\left[\delta F /\left(\delta U_{j}\right)\right]=\left[\partial F /\left(\partial U_{j}\right)\right]-[d /(d x)]$ $\left[\partial F /\left(\partial U_{j}^{\prime}\right)\right]$, with $\varepsilon_{i j k}$ the antisymmetric tensor. Here and below, the prime denotes the spatial partial derivative; e.g., $U_{i}^{\prime}=\partial_{x} U_{i}$. In particular, we have $\left\{U_{i}(x)\right.$, $\left.U_{j}\left(x^{\prime}\right)\right\}=\varepsilon_{i j k} U_{k} \delta\left(x-x^{\prime}\right),\left\{V_{i}(x), V_{j}\left(x^{\prime}\right)\right\}=\varepsilon_{i j k} V_{k} \delta\left(x-x^{\prime}\right)$. Equations (25) and (26) can then be written as $\partial_{t} \boldsymbol{U}=\{\boldsymbol{U}, \mathcal{H}\}, \partial_{t} \boldsymbol{V}=\{\boldsymbol{V}, \mathcal{H}\}$, with the Hamiltonian $\mathcal{H}=\mathcal{H}_{U}+\mathcal{H}_{V}+\mathcal{H}_{\text {int }}$, explicitly given in Eq. (27). Making use of $\boldsymbol{U} \cdot \boldsymbol{U}^{\prime}=0, \boldsymbol{V} \cdot \boldsymbol{V}^{\prime}=0$, we have $\boldsymbol{U}^{\prime}=\left\{\boldsymbol{U}, P_{U}\right\}, \boldsymbol{V}^{\prime}=\left\{\boldsymbol{V}, P_{V}\right\}$, where the momenta read

$$
\begin{gathered}
P_{U}=\frac{U_{2}}{U_{0}^{2}-U_{2}^{2}}\left(U_{3} U_{1}^{\prime}-U_{1} U_{3}^{\prime}\right), \\
P_{V}=\frac{V_{2}}{V_{0}^{2}-V_{2}^{2}}\left(V_{3} V_{1}^{\prime}-V_{1} V_{3}^{\prime}\right),
\end{gathered}
$$

and the conserved total momentum is $\mathcal{P}=\int P_{U}+P_{V} d x$.

\section{Cylindrical coordinates}

It proves convenient to rewrite the Hamiltonian in cylindrical coordinates. The canonical cylindrical coordinates $\left(U_{2}, \alpha_{U}, V_{2}, \alpha_{V}\right)$ are defined by

$$
\begin{aligned}
& \boldsymbol{U}=\left[\sqrt{U_{0}^{2}-U_{2}^{2}} \sin \left(\alpha_{U}\right), U_{2}, \sqrt{U_{0}^{2}-U_{2}^{2}} \cos \left(\alpha_{U}\right)\right], \\
& \boldsymbol{V}=\left[\sqrt{V_{0}^{2}-V_{2}^{2}} \sin \left(\alpha_{V}\right), V_{2}, \sqrt{V_{0}^{2}-V_{2}^{2}} \cos \left(\alpha_{V}\right)\right] .
\end{aligned}
$$

In these coordinates, the corresponding canonical equations read:

$$
\begin{gathered}
\left(\partial_{t}+w \partial_{x}\right) \alpha_{U}=\partial H_{s} / \partial U_{2}, \\
\left(\partial_{t}+w \partial_{x}\right) U_{2}=-\partial H_{s} / \partial \alpha_{U}, \\
\left(\partial_{t}-w \partial_{x}\right) \alpha_{V}=\partial H_{s} / \partial V_{2}, \\
\left(\partial_{t}-w \partial_{x}\right) V_{2}=-\partial H_{s} / \partial \alpha_{V},
\end{gathered}
$$

where $H_{s}=-\frac{1}{2}(\boldsymbol{U}+\boldsymbol{V}) \cdot \mathcal{I}(\boldsymbol{U}+\boldsymbol{V})=-\kappa\left[\sqrt{U_{0}^{2}-U_{2}^{2}} \times\right.$ $\left.\sqrt{V_{0}^{2}-V_{2}^{2}} \cos \left(\alpha_{U}-\alpha_{V}\right)+U_{2} V_{2}\right]-(1-\kappa)\left(U_{2}+V_{2}\right)^{2}-$ $\frac{\kappa}{2}\left(U_{0}^{2}+V_{0}^{2}\right)$. These equations are equivalent to Eqs. (24) and (25). The advantage of these canonical cylindrical coordinates with respect to the spherical Stokes parameters is that the momentum contribution to the Hamiltonian takes a much simpler form. Using the fact that $P_{U}=U_{2} \alpha_{U}^{\prime}$ and $P_{V}=V_{2} \alpha_{V}^{\prime}$, the Hamiltonian has the form $\mathcal{H}=$ $\int \hat{\mathcal{H}}\left(U_{2}, V_{2}, \alpha_{U}, \alpha_{V}, \alpha_{U}^{\prime}, \alpha_{V}^{\prime}\right) d x$, with 


$$
\begin{aligned}
\hat{\mathcal{H}}\left(U_{2}, V_{2}, \alpha_{U}, \alpha_{V}, \alpha_{U}^{\prime}, \alpha_{V}^{\prime}\right) & \\
= & w\left(V_{2} \alpha_{V}^{\prime}-U_{2} \alpha_{U}^{\prime}\right) \\
& -\kappa\left[\sqrt{U_{0}^{2}-U_{2}^{2}} \sqrt{V_{0}^{2}-V_{2}^{2}} \cos \left(\alpha_{U}-\alpha_{V}\right)+U_{2} V_{2}\right] \\
& -(1-\kappa)\left(U_{2}+V_{2}\right)^{2}-\frac{\kappa}{2}\left(U_{0}^{2}+V_{0}^{2}\right),
\end{aligned}
$$

the momentum reads $\mathcal{P}=\int \hat{\mathcal{P}}\left(U_{2}, V_{2}, \alpha_{U}, \alpha_{V}, \alpha_{U}^{\prime}, \alpha_{V}^{\prime}\right) d x$, with

$$
\hat{\mathcal{P}}\left(U_{2}, V_{2}, \alpha_{U}, \alpha_{V}, \alpha_{U}^{\prime}, \alpha_{V}^{\prime}\right)=U_{2} \alpha_{U}^{\prime}+V_{2} \alpha_{V}^{\prime},
$$

and the magnetization reads $\mathcal{M}=\int \hat{\mathcal{M}}\left(U_{2}, V_{2}, \alpha_{U}, \alpha_{V}\right.$, $\left.\alpha_{U}^{\prime}, \alpha_{V}^{\prime}\right) d x$, with

$$
\hat{\mathcal{M}}\left(U_{2}, V_{2}, \alpha_{U}, \alpha_{V}, \alpha_{U}^{\prime}, \alpha_{V}^{\prime}\right)=-\frac{1}{2}\left(U_{2}+V_{2}\right) .
$$

\section{APPENDIX D: DERIVATION OF THE PROBABILITY DENSITIES [EQS. (30)-(35)]}

\section{General expression}

We begin by introducing a finite-dimensional approximation of the partial differential equation (C6)-(C9) through a spatial discretization of the variable $x$, which gives a finite-dimensional system governing the evolution of $\left(\boldsymbol{K}_{j}\right)_{j=1}^{n}$, where $\boldsymbol{K}=\left(U_{2}, V_{2}, \alpha_{U}, \alpha_{V}, \alpha_{U}^{\prime}, \alpha_{V}^{\prime}\right)$ is an element of the phase space. The equilibrium density $\rho$ maximizes the Gibbs entropy functional, $\mathcal{S}_{G}[\rho]=$ $-\int \rho\left[\left(\boldsymbol{K}_{j}\right)_{j=1}^{n}\right] \log \left\{\rho\left[\left(\boldsymbol{K}_{j}\right)_{j=1}^{n}\right]\right\} d \boldsymbol{K}_{1} \ldots d \boldsymbol{K}_{n}$, under the constraints dictated by the three conserved quantities, the Hamiltonian $\mathcal{H}$ [Eq. (C10)], the momentum $\mathcal{P}$ [Eq. (C11)], and the magnetization $\mathcal{M}$ [Eq. (C12)]. Following the standard procedure based on the Lagrangian multipliers, the equilibrium PDF reads $\rho\left[\left(\boldsymbol{K}_{j}\right)_{j=1}^{n}\right] \sim$ $\exp \left\{-\beta \mathcal{H}\left[\left(\boldsymbol{K}_{j}\right)_{j=1}^{n}\right]-\nu \mathcal{P}\left[\left(\boldsymbol{K}_{j}\right)_{j=1}^{n}\right]-\gamma \mathcal{M}\left[\left(\boldsymbol{K}_{j}\right)_{j=1}^{n}\right]\right\}$. In the long-term evolution, the waves $\boldsymbol{U}(x, t)$ and $\boldsymbol{V}(x, t)$ exhibit fluctuations that are statistically homogeneous in space, with a correlation length that decreases during the evolution. In this way, the large convection among the waves $(w \gg 1)$ leads to an effective averaging of the spatial correlations of the waves, so that the state of maximum entropy $\rho\left[\left(\boldsymbol{K}_{j}\right)_{j=1}^{n}\right] \sim$ $\exp \left[-\beta \sum_{j=1}^{n} \hat{\mathcal{H}}\left(\boldsymbol{K}_{j}\right)-\nu \sum_{j=1}^{n} \hat{\mathcal{P}}\left(\boldsymbol{K}_{j}\right)-\gamma \sum_{j=1}^{n} \hat{\mathcal{M}}\left(\boldsymbol{K}_{j}\right)\right]$, with $\hat{\mathcal{H}}, \hat{\mathcal{P}}$, and $\hat{\mathcal{M}}$ given by Eqs. (C10)-(C12), results to be the product of the elementary densities $\prod_{j=1}^{n} \rho\left(\boldsymbol{K}_{j}\right)$, with

$$
\rho(\boldsymbol{K}) \sim \exp [-\beta \hat{\mathcal{H}}(\boldsymbol{K})-\nu \hat{\mathcal{P}}(\boldsymbol{K})-\gamma \hat{\mathcal{M}}(\boldsymbol{K})],
$$

where $\boldsymbol{K}$ stands for $\boldsymbol{K}_{j}$ to simplify the notations.

\section{Reduced form of the equilibrium density}

According to Eq. (31), we have $\nu=\beta=0$, and we now look for the equilibrium PDF distribution $\rho(\boldsymbol{Q})$, by considering solely the constraint imposed by the conservation of $\mathcal{M}$. The vector $\boldsymbol{Q}=(\boldsymbol{U}, \boldsymbol{V})$ is an element of the phase space that refers to the product of the two spheres of radii $U_{0}$ and $V_{0}$. We refer back to the original variables so as to derive the equilibrium PDF in terms of the correlators $\left(n_{\varphi_{i}}^{0}, m_{\mu}^{0}\right)$. Accordingly, the vectors $\left[\tilde{n}_{\varphi_{p}}^{0}, \operatorname{Re}\left(m_{\mu}^{0}\right), \operatorname{Im}\left(m_{\mu}^{0}\right)\right]$ evolve on the surface of two spheres of radii $N_{\varphi} / 2$, with $(\varphi=u, \mu=u v)$ or $(\varphi=v, \mu=v u)$. We represent these vectors in spherical coordinates: $\tilde{n}_{\varphi_{p}}^{0}=\left(N_{\varphi} / 2\right) \cos \theta_{\varphi}$ $(\varphi=u, \quad v) ; \quad \operatorname{Re}\left(m_{\mu}^{0}\right)=\left(N_{\varphi} / 2\right) \sin \theta_{\varphi} \cos \delta_{\varphi}, \quad \operatorname{Im}\left(m_{\mu}^{0}\right)=$ $\left(N_{\varphi} / 2\right) \sin \theta_{\varphi} \sin \delta_{\varphi}$, with $(\varphi=u, \mu=u v)$ or $(\varphi=v, \mu=v u)$. We look for the stationary distribution of the state vector $\left(\theta_{u}, \delta_{u}, \theta_{v}, \delta_{v}\right)$ by maximizing the entropy under the constraint imposed by the conservation of the magnetization $\mathcal{M}$, which can be written as $L^{-1} \int_{0}^{L}\left[\tilde{n}_{u_{p}}^{0}(x, t)-\tilde{n}_{v_{p}}^{0}(x, t)\right] d x=$ $\left(N_{v}-N_{u}\right) / 2$. Following the usual procedure based on the method of the Lagrange multipliers, we obtain the PDF of the equilibrium distribution:

$\rho\left(\theta_{u}, \delta_{u}, \theta_{v}, \delta_{v}\right)=\mathcal{Z}^{-1} \exp \left[\gamma\left(\frac{N_{u}}{2} \cos \theta_{u}-\frac{N_{v}}{2} \cos \theta_{v}\right)\right]$,

where the multiplier $\gamma$, associated to the conservation of $\mathcal{M}$, is the unique solution of

$$
-\frac{2}{\gamma}+\frac{N_{u}}{2} \frac{1}{\tanh \left(\gamma N_{u} / 2\right)}+\frac{N_{v}}{2} \frac{1}{\tanh \left(\gamma N_{v} / 2\right)}=\frac{N_{v}-N_{u}}{2},
$$

and $\mathcal{Z}$ is given by

$$
\mathcal{Z}=\frac{64 \pi^{2}}{N_{u} N_{v} \gamma^{2}} \sinh \left(\frac{\gamma N_{u}}{2}\right) \sinh \left(\frac{\gamma N_{v}}{2}\right) .
$$

The PDF Eq. (D2) shows, in particular, that $\left[\tilde{n}_{u_{p}}^{0}, \operatorname{Re}\left(m_{u v}^{0}\right)\right.$, $\left.\operatorname{Im}\left(m_{u v}^{0}\right)\right]$ and $\left[\tilde{n}_{v_{p}}^{0}, \operatorname{Re}\left(m_{v u}^{0}\right), \operatorname{Im}\left(m_{v u}^{0}\right)\right]$ are independent. The marginal PDFs for the normal correlator $p\left(n_{\varphi_{j}}^{0}\right)$ and anomalous correlator $p\left(\left|m_{\mu}^{0}\right|\right)$ given in Eqs. (34) and (35) have been computed from the general expression of the PDF Eq. (D2).

\section{APPENDIX E: HAMILTONIAN STRUCTURE OF THE THIRRING AND THREE-WAVE INTERACTION MODELS}

In order to illustrate the generality of the process of unconstrained thermalization, here, we show that the phasecorrelation kinetic equations (24) and (25) [or Eqs. (20) and (21)] exhibit a Hamiltonian structure analogous to that of the Thirring model and the three-wave interaction model. The generalized massive Thirring model can be recast in the following dimensionless form $[91,92,94,95]$ :

$$
\begin{gathered}
i \partial_{t} u+i \partial_{x} u=-\delta v-\left(|u|^{2}+2|v|^{2}\right) u, \\
i \partial_{t} v-i \partial_{x} v=-\delta u-\left(|v|^{2}+2|u|^{2}\right) v,
\end{gathered}
$$


where $u$ and $v$ denote the forward and backward wave amplitudes, while $\delta$ is the linear coupling coefficient of the structure. These equations conserve the total energy $N=\int|u|^{2}+|v|^{2} d x$, the Hamiltonian $H=\int P_{v}-P_{u}-$ $\frac{1}{2}\left(|u|^{4}+|v|^{4}\right)-2|u|^{2}|v|^{2}-\kappa\left(u v^{*}+v u^{*}\right) d x$, and the momentum $P=\int P_{v}+P_{u} d x$, with $P_{\varphi}=\operatorname{Im}\left(\varphi \partial_{x} \varphi^{*}\right)$, $\varphi=u, v$. On the other hand, the equations for the threewave interaction can be written in the form [96]

$$
\begin{gathered}
i \partial_{t} u_{1}+i w_{1} \partial_{x} u_{1}=-u_{3} u_{2}^{*}, \\
i \partial_{t} u_{2}+i w_{2} \partial_{x} u_{2}=-u_{3} u_{1}^{*}, \\
i \partial_{t} u_{3}=-u_{1} u_{2},
\end{gathered}
$$

where $w_{j}$ denote the velocity differences between the daughter waves $u_{1,2}$ and the pump wave $u_{3}$. These equations conserve the Manley-Rowe invariants for the energies $N_{1}=\int\left|u_{1}\right|^{2}+\left|u_{3}\right|^{2} d x, \quad N_{2}=\int\left|u_{2}\right|^{2}+\left|u_{3}\right|^{2} d x$, the Hamiltonian $H=\int-w_{1} P_{1}-w_{2} P_{2}-u_{1} u_{2} u_{3}^{*}-u_{1}^{*} u_{2}^{*} u_{3} d x$, and the total momentum $P=\int P_{1}+P_{2}+P_{3} d x$, with $P_{j}=\operatorname{Im}\left(u_{j} \partial_{x} u_{j}^{*}\right)$. Note that the signs of the velocities $\left(w_{j}\right)$ of the three waves can be changed by simply writing the equations in different inertial references frames.

It becomes apparent from the expressions of the conserved quantities that the generalized massive Thirring model and the resonant three-wave interaction model exhibit a Hamiltonian structure analogous to that of the phase-correlation kinetic equations [see the relation between the Hamiltonian and the momenta in Eq. (27)]. Then, following the same reasoning as that developed in Sec. IV C, one arrives at the conclusion that the Hamiltonian and the momentum do not constrain the maximization of the Gibbs entropy at equilibrium.

[1] E. Fermi, J. Pasta, and S. Ulam, Los Alamos Scientific Laboratory Report No. LA-1940, 1955.

[2] J. Ford, The Fermi-Pasta-Ulam Problem: Paradox Turns Discovery, Phys. Rep. 213, 271 (1992).

[3] Focus issue on The "Fermi-Pasta-Ulam" Problem-The First 50 years, Chaos 15, No. 1 (2005).

[4] The Fermi-Pasta-Ulam Problem: A Status Report, edited by G. Gallavotti (Springer, New York, 2007).

[5] R. Livi, M. Pettini, S. Ruffo, M. Sparpaglione, and A. Vulpiani, Equipartition Threshold in Nonlinear Large Hamiltonian Systems: The Fermi-Pasta-Ulam Model, Phys. Rev. A 31, 1039 (1985).

[6] G. P. Berman and F. M. Izrailev, The Fermi-Pasta-Ulam Problem: Fifty Years of Progress, Chaos 15, 015104 (2005).

[7] B. Gershgorin, Y. V. L'vov, and D. Cai, Renormalized Waves and Discrete Breathers in B-Fermi-Pasta-Ulam Chains, Phys. Rev. Lett. 95, 264302 (2005).
[8] B. Gershgorin, Y. V. L'vov, and D. Cai, Interactions of Renormalized Waves in Thermalized Fermi-Pasta-Ulam Chains, Phys. Rev. E 75, 046603 (2007).

[9] S. W. Jiang, H. Lu, D. Zhou, and D. Cai, Renormalized Dispersion Relations of $\beta$-Fermi-Pasta-Ulam Chains in Equilibrium and Nonequilibrium States, Phys. Rev. E 90, 032925 (2014).

[10] G. Benettin, H. Christodoulidi, and A. Ponno, The Fermi-Pasta-Ulam Problem and Its Underlying Integrable Dynamics, J. Stat. Phys. 152, 195 (2013).

[11] M. Onorato, L. Vozella, D. Proment, and Y. V. L'vov, Route to Thermalization in the $\alpha$-Fermi-Pasta-Ulam System, Proc. Natl. Acad. Sci. U.S.A. 112, 4208 (2015).

[12] E. A. Kuznetsov, Fermi-Pasta-Ulam Recurrence and Modulation Instability, arXiv:1605.05080.

[13] N. J. Zabusky and M. D. Kruskal, Interaction of "Solitons" in a Collisionless Plasma and the Recurrence of Initial States, Phys. Rev. Lett. 15, 240 (1965).

[14] N. Akhmediev and V. Korneev, Modulation Instability and Periodic Solutions of the Nonlinear Schrödinger Equation, Theor. Math. Phys. 69, 1089 (1986).

[15] V. E. Zakharov and E. I. Schulman, What Is Integrability? (Springer, Berlin, 1991).

[16] B. M. Lake, H. C. Yuen, H. Rungaldier, and W. E. Ferguson, Nonlinear Deep-Water Waves: Theory and Experiment. Part 2. Evolution of a Continuous Wave Train, J. Fluid Mech. 83, 49 (1977).

[17] M. Wu and C.E. Patton, Experimental Observation of Fermi-Pasta-Ulam Recurrence in a Nonlinear Feedback Ring System, Phys. Rev. Lett. 98, 047202 (2007).

[18] G. Simaeys, P. Emplit, and M. Haelterman, Experimental Demonstration of the Fermi-Pasta-Ulam Recurrence in a Modulationally Unstable Optical Wave, Phys. Rev. Lett. 87, 033902 (2001).

[19] J. Beeckman, X. Hutsebaut, M. Haelterman, and K. Neyts, Induced Modulation Instability and Recurrence in Nematic Liquid Crystals, Opt. Express 15, 11185 (2007).

[20] A. Mussot, A. Kudlinski, M. Droques, P. Szriftgiser, and N. Akhmediev, Fermi-Pasta-Ulam Recurrence in Nonlinear Fiber Optics: The Role of Reversible and Irreversible Losses, Phys. Rev. X 4, 011054 (2014).

[21] M. Onorato, S. Residori, U. Bortolozzo, A. Montina, and F. T. Arecchi, Rogue Waves and Their Generating Mechanisms in Different Physical Contexts, Phys. Rep. 528, 47 (2013).

[22] J. M. Dudley, F. Dias, M. Erkintalo, and G. Genty, Instabilities, Breathers and Rogue Waves in Optics, Nat. Photonics 8, 755 (2014).

[23] E. A. Kuznetsov, Solitons in a Parametrically Unstable Plasma, Sov. Phys. Dokl. 22, 507 (1977).

[24] Y.C. Ma, The Perturbed Plane-Wave Solutions of the Cubic Schrödinger Equation, Stud. Appl. Math. 60, 43 (1979).

[25] N. Akhmediev, V. M. Eleonskii, and N. E. Kulagin, Exact First-Order Solutions of the Nonlinear Schrödinger Equation, Theor. Math. Phys. 72, 809 (1987).

[26] B. Kibler, J. Fatome, C. Finot, G. Millot, F. Dias, G. Genty, N. Akhmediev, and J. M. Dudley, The Peregrine Soliton in Nonlinear Fibre Optics, Nat. Phys. 6, 790 (2010). 
[27] M. Erkintalo, K. Hammani, B. Kibler, C. Finot, N. Akhmediev, J. M. Dudley, and G. Genty, Higher-Order Modulation Instability in Nonlinear Fiber Optics, Phys. Rev. Lett. 107, 253901 (2011).

[28] S. Toenger, T. Godin, C. Billet, F. Dias, M. Erkintalo, G. Genty, and J. M. Dudley, Emergent Rogue Wave Structures and Statistics in Spontaneous Modulation Instability, Sci. Rep. 5, 10380 (2015).

[29] A. Chabchoub, N. P. Hoffmann, and N. Akhmediev, Rogue Wave Observation in a Water Wave Tank, Phys. Rev. Lett. 106, 204502 (2011).

[30] A. Chabchoub, N. Hoffmann, M. Onorato, and N. Akhmediev, Super Rogue Waves: Observation of a Higher-Order Breather in Water Waves, Phys. Rev. X 2, 011015 (2012).

[31] F. Baronio, A. Degasperis, M. Conforti, and S. Wabnitz, Solutions of the Vector Nonlinear Schrödinger Equations: Evidence for Deterministic Rogue Waves, Phys. Rev. Lett. 109, 044102 (2012).

[32] F. Baronio, M. Conforti, A. Degasperis, S. Lombardo, M. Onorato, and S. Wabnitz, Vector Rogue Waves and Baseband Modulation Instability in the Defocusing Regime, Phys. Rev. Lett. 113, 034101 (2014).

[33] V. E. Zakharov and A. A. Gelash, Nonlinear Stage of Modulation Instability, Phys. Rev. Lett. 111, 054101 (2013).

[34] B. Kibler, A. Chabchoub, A. Gelash, N. Akhmediev, and V.E. Zakharov, Super-regular Breathers in Optics and Hydrodynamics: Omnipresent Modulation Instability Beyond Simple Periodicity, Phys. Rev. X 5, 041026 (2015).

[35] O. Kimmoun, H. C. Hsu, H. Branger, M. S. Li, Y. Y. Chen, C. Kharif, M. Onorato, E. J. R. Kelleher, B. Kibler, N. Akhmediev, and A. Chabchoub, Modulation Instability and Phase-Shifted Fermi-Pasta-Ulam Recurrence, Sci. Rep. 6, 28516 (2016).

[36] V. E. Zakharov, V. S. L'vov, and G. Falkovich, Kolmogorov Spectra of Turbulence I (Springer, Berlin, 1992).

[37] V.S. L'vov, Wave Turbulence Under Parametric Excitation-Application to Magnets (Springer, Berlin, 1994).

[38] V. Zakharov, F. Dias, and A. Pushkarev, One-Dimensional Wave Turbulence, Phys. Rep. 398, 1 (2004).

[39] S. Nazarenko, Wave Turbulence, Lecture Notes in Physics (Springer, New York, 2011).

[40] A. C. Newell and B. Rumpf, Wave Turbulence, Annu. Rev. Fluid Mech. 43, 59 (2011).

[41] Advances in Wave Turbulence, World Scientific Series on Nonlinear Science Series A, Vol. 83, edited by V. I. Shrira and S. Nazarenko (World Scientific, Singapore, 2013).

[42] V. S. L'vov, Y. V. Lvov, A. C. Newell, and V. E. Zakharov, Statistical Description of Acoustic Turbulence, Phys. Rev. E 56, 390 (1997).

[43] B. Rumpf, A. C. Newell, and V. E. Zakharov, Turbulent Transfer of Energy by Radiating Pulses, Phys. Rev. Lett. 103, 074502 (2009).

[44] A. Newell, B. Rumpf, and V. Zakharov, Spontaneous Breaking of the Spatial Homogeneity Symmetry in Wave Turbulence, Phys. Rev. Lett. 108, 194502 (2012).

[45] J. Laurie, U. Bortolozzo, S. Nazarenko, and S. Residori, One-Dimensional Optical Wave Turbulence: Experiment and Theory, Phys. Rep. 514, 121 (2012).
[46] A. Picozzi, J. Garnier, T. Hansson, P. Suret, S. Randoux, G. Millot, and D. Christodoulides, Optical Wave Turbulence: Toward a Unified Nonequilibrium Thermodynamic Formulation of Statistical Nonlinear Optics, Phys. Rep. 542, 1 (2014).

[47] S. K. Turitsyn, S. A. Babin, E. G. Turitsyna, G. E. Falkovich, E. Podivilov, and D. Churkin, Optical Wave Turbulence, in Advances in Wave Turbulence, edited by V. I. Shira and S. Nazarenko, World Scientific Series on Nonlinear Science Series A, Vol. 83 (World Scientific, Singapore, 2013).

[48] D. Churkin, I. Kolokolov, E. Podivilov, I. Vatnik, S. Vergeles, I. Terekhov, V. Lebedev, G. Falkovich, M. Nikulin, S. Babin, and S. Turitsyn, Wave Kinetics of a Random Fibre Laser, Nat. Commun. 2, 6214 (2015).

[49] A. Fratalocchi, C. Conti, G. Ruocco, and S. Trillo, FreeEnergy Transition in a Gas of Noninteracting Nonlinear Wave Particles, Phys. Rev. Lett. 101, 044101 (2008).

[50] F. Antenucci, M. Ibanez Berganza, and L. Leuzzi, Statistical Physics of Nonlinear Wave Interaction, Phys. Rev. B 92, 014204 (2015).

[51] Y. Silberberg, Y. Lahini, Y. Bromberg, E. Small, and R. Morandotti, Universal Correlations in a Nonlinear Periodic 1D System, Phys. Rev. Lett. 102, 233904 (2009).

[52] A. Picozzi, Nonequilibrated Oscillations of Coherence in Coupled Nonlinear Wave Systems, Phys. Rev. Lett. 96, 013905 (2006).

[53] N. Vladimirova, S. Derevyanko, and G. Falkovich, Phase Transitions in Wave Turbulence, Phys. Rev. E 85, 010101 (R) (2012).

[54] S. A. Derevyanko, Thermalized Polarization Dynamics of a Discrete Optical-Waveguide System with Four-Wave Mixing, Phys. Rev. A 88, 033851 (2013).

[55] B. Fischer and A. Bekker, Many Body Photonics, Opt. Photonics News 24, 40 (2013).

[56] S. Wabnitz, A. Picozzi, A. Tonello, D. Modotto, and G. Millot, Control of Signal Coherence in Parametric Frequency Mixing with Incoherent Pumps, J. Opt. Soc. Am. B 29, 3128 (2012).

[57] F. Antenucci, C. Conti, A. Crisanti, and L. Leuzzi, General Phase Diagram of Multimodal Ordered and Disordered Lasers in Closed and Open Cavities, Phys. Rev. Lett. 114, 043901 (2015).

[58] Y. Bromberg, Y. Lahini, E. Small, and Y. Silberberg, Hanbury Brown and Twiss Interferometry with Interacting Photons, Nat. Photonics 4, 721 (2010).

[59] P. Miller, N. Vladimirova, and G. Falkovich, Oscillations in a Turbulence-Condensate System, Phys. Rev. E 87, 065202 (2013).

[60] G. Düring, A. Picozzi, and S. Rica, Breakdown of Weak-Turbulence and Nonlinear Wave Condensation, Physica (Amsterdam) 238D, 1524 (2009).

[61] E. Turitsyna, S. Smirnov, S. Sugavanam, N. Tarasov, X. Shu, S. Babin, E. Podivilov, D. Churkin, G. Falkovich, and S. Turitsyn, The Laminar-Turbulent Transition in a Fibre Laser, Nat. Photonics 7, 783 (2013).

[62] B. Miquel, A. Alexakis, C. Josserand, and, N. Mordant, Transition from Wave Turbulence to Dynamical Crumpling in Vibrated Elastic Plates, Phys. Rev. Lett. 111, 054302 (2013). 
[63] G. Xu, D. Vocke, D. Faccio, J. Garnier, T. Rogers, S. Trillo, and A. Picozzi, From Coherent Shocklets to Giant Collective Incoherent Shock Waves in Nonlocal Turbulent Flows, Nat. Commun. 6, 8131 (2015).

[64] A. Picozzi and J. Garnier, Incoherent Soliton Turbulence in Nonlocal Nonlinear Media, Phys. Rev. Lett. 107, 233901 (2011).

[65] C. Conti, M. Leonetti, A. Fratalocchi, L. Angelani, and G. Ruocco, Condensation in Disordered Lasers: Theory, Simulations, and Experiments, Phys. Rev. Lett. 101, 143901 (2008).

[66] A. Ribal, A. V. Babanin, I. Young, A. Toffoli, and M. Stiassnie, Recurrent Solutions of the Alber Equation Initialized by Joint North Sea Wave Project Spectra, J. Fluid Mech. 719, 314 (2013).

[67] E. Infeld and G. Rowlands, Nonlinear Waves, Solitons and Chaos (Cambridge University Press, Cambridge, 1990).

[68] P. A. E. M. Janssen, Long-Time Behaviour of a Random Inhomogeneous Field of Weakly Nonlinear Surface Gravity Waves, J. Fluid Mech. 133, 113 (1983).

[69] B. Hall, M. Lisak, D. Anderson, R. Fedele, and V.E. Semenov, Statistical Theory for Incoherent Light Propagation in Nonlinear Media, Phys. Rev. E 65, 035602(R) (2002).

[70] M. Onorato, A. Osborne, R. Fedele, and M. Serio, Landau Damping and Coherent Structures in Narrow-Banded $1+1$ Deep Water Gravity Waves, Phys. Rev. E 67, 046305 (2003).

[71] P. A. E. M. Janssen, Nonlinear Four-Wave Interactions and Freak Waves, J. Phys. Oceanogr. 33, 863 (2003).

[72] P. A. E. M. Janssen, The Interaction of Ocean Waves and Wind (Cambridge University Press, Cambridge, 2004).

[73] L. Landau and E. Lifshitz, Statistical Physics (Pergamon, New York, 1980).

[74] Y. Kivshar and G. P. Agrawal, Optical Solitons: From Fibers to Photonic Crystals (Academic, New York, 2003).

[75] C. Mc Kinstrie and D. Russell, Nonlinear Focusing of Coupled Waves, Phys. Rev. Lett. 61, 2929 (1988).

[76] M. Onorato, A. R. Osborne, and M. Serio, Modulational Instability in Crossing Sea States: A Possible Mechanism for the Formation of Freak Waves, Phys. Rev. Lett. 96, 014503 (2006).

[77] J. M. Bilbault, P. Marquie, and B. Michaux, Modulational Instability of Two Counterpropagating Waves in an Experimental Transmission Line, Phys. Rev. E 51, 817 (1995).

[78] L. Pitaevskii and S. Stringari, Bose-Einstein Condensation (Oxford Science Publications, New York, 2003).

[79] D. J. Kaup, A Perturbation Expansion for the ZakharovShabat Inverse Scattering Transform, SIAM J. Appl. Math. 31, 121 (1976).

[80] V.S. Shchesnovich and E. V. Doktorov, Perturbation Theory for Solitons of the Manakov System, Phys. Rev. E 55, 7626 (1997).

[81] T. I. Lakoba and D. J. Kaup, Perturbation Theory for the Manakov Soliton and Its Applications to Pulse Propagation in Randomly Birefringent Fibers, Phys. Rev. E 56, 6147 (1997).

[82] M. Soljacic, M. Segev, T. Coskun, D. N. Christodoulides, and A. Vishwanath, Modulation Instability of Incoherent
Beams in Noninstantaneous Nonlinear Media, Phys. Rev. Lett. 84, 467 (2000).

[83] D. Kip, M. Soljacic, M. Segev, E. Eugenieva, and D. N. Christodoulides, Modulation Instability and Pattern Formation in Spatially Incoherent Light Beams, Science 290, 495 (2000).

[84] M. Segev and D. N. Christodoulides, in Spatial Solitons, edited by S. Trillo and W. Torruellas (Springer, Berlin, 2001).

[85] M. Onorato, A. R. Osborne, M. Serio, L. Cavaleri, C. Brandini, and C. T. Stansberg, Observation of Strongly Non-Gaussian Statistics for Random Sea Surface Gravity Waves in Wave Flume Experiments, Phys. Rev. E 70, 067302 (2004).

[86] M. Onorato, A. R. Osborne, M. Serio, and L. Cavaleri, Modulational Instability and Non-Gaussian Statistics in Experimental Random Water-Wave Trains, Phys. Fluids 17, 078101 (2005).

[87] D. David, D. D. Holm, and M. V. Tratnik, Hamiltonian Chaos in Nonlinear Optical Polarization Dynamics, Phys. Rep. 187, 281 (1990).

[88] S. Pitois, G. Millot, and S. Wabnitz, Polarization Domain Wall Solitons with Counterpropagating Laser Beams, Phys. Rev. Lett. 81, 1409 (1998).

[89] J. Fatome, S. Pitois, P. Morin, E. Assémat, D. Sugny, A. Picozzi, H. R. Jauslin, G. Millot, V. V. Kozlov, and S. Wabnitz, A Universal Optical All-Fiber Omnipolarizer, Sci. Rep. 2, 938 (2012).

[90] We split the nonlinear energy into the self- and the crossinteraction contributions, $\mathcal{H}_{s}=-\frac{1}{2} \int(\boldsymbol{U}+\boldsymbol{V}) \cdot \mathcal{I}(\boldsymbol{U}+\boldsymbol{V}) d x=$ $\mathcal{U}_{U}+\mathcal{U}_{V}+\mathcal{H}_{\text {int }}, \quad$ with $\quad \mathcal{U}_{U} \simeq-\frac{1}{2} \sum_{j=1}^{3} \nu_{j}\left\langle U_{j}^{2}\right\rangle, \quad \mathcal{U}_{V} \simeq$ $-\frac{1}{2} \sum_{j=1}^{3} \nu_{j}\left\langle V_{j}^{2}\right\rangle$, and $\mathcal{H}_{\text {int }} \simeq-\sum_{j=1}^{3} \nu_{j}\left\langle U_{j} V_{j}\right\rangle$, with $\nu_{j}$ the three terms of the diagonal matrix $\mathcal{I}$. Because of large convection $(w \gg 1)$, the correlations among $\boldsymbol{U}$ and $\boldsymbol{V}$ are averaged out, $\mathcal{H}_{\text {int }} \ll \mathcal{U}_{U, V}$. This has been confirmed by the numerical simulations near the equilibrium state.

[91] D. N. Christodoulides and R. I. Joseph, Slow Bragg Solitons in Nonlinear Periodic Structures, Phys. Rev. Lett. 62, 1746 (1989).

[92] G. Lenz, P. Meystre, and E. M. Wright, Nonlinear Atom Optics, Phys. Rev. Lett. 71, 3271 (1993).

[93] O. Morsch and M. Oberthaler, Dynamics of Bose-Einstein Condensates in Optical Lattices, Rev. Mod. Phys. 78, 179 (2006).

[94] A. Degasperis, S. Wabnitz, and A. B. Aceves, Bragg Grating Rogue Wave, Phys. Lett. A 379, 1067 (2015).

[95] V.P. Ruban, Water-Wave Gap Solitons: An Approximate Theory and Numerical Solutions of the Exact Equations of Motion, Phys. Rev. E 78, 066308 (2008).

[96] D. J. Kaup, A. Reiman, and A. Bers, Space-Time Evolution of Nonlinear Three-Wave Interactions. I. Interaction in a Homogeneous Medium, Rev. Mod. Phys. 51, 275 (1979).

[97] A. M. Balk and E. V. Ferapontov, in Nonlinear Waves and Weak Turbulence, edited by V. E. Zakharov (American Mathematical Society, Providence, 1997).

[98] G. A. El and A. M. Kamchatnov, Kinetic Equation for a Dense Soliton Gas, Phys. Rev. Lett. 95, 204101 (2005).

[99] P. Suret, S. Randoux, H. R. Jauslin, and A. Picozzi, Anomalous Thermalization of Nonlinear Wave Systems, Phys. Rev. Lett. 104, 054101 (2010). 
[100] S. Y. Annenkov and V. I. Shrira, Role of Non-Resonant Interactions in the Evolution of Nonlinear Random Water Wave Fields, J. Fluid Mech. 561, 181 (2006).

[101] P. Suret, A. Picozzi, and S. Randoux, Wave Turbulence in Integrable Systems: Nonlinear Propagation of Incoherent Optical Waves in Single-Mode Fibers, Opt. Express 19, 17852 (2011).

[102] L. Deng, E. W. Hagley, J. Wen, M. Trippenbach, Y. Band, P. S. Julienne, J. E. Simsarian, K. Helmerson, S. L. Rolston, and W. D. Phillips, Four-Wave Mixing with Matter Waves, Nature (London) 398, 218 (1999).

[103] M. C. Tsatsos, P. E. S. Tavares, A. Cidrim, A. R. Fritsch, M. A. Caracanhas, F. A. dos Santos, C. F. Barenghi, and V.S. Bagnato, Quantum Turbulence in Trapped Atomic Bose-Einstein Condensates, Phys. Rep. 622, 1 (2016).

[104] N. Akhmediev, A. Ankiewicz, and J. M. Soto-Crespo, Rogue Waves and Rational Solutions of the Nonlinear
Schrödinger Equation, Phys. Rev. E 80, 026601 (2009).

[105] P. Walczak, S. Randoux, and P. Suret, Optical Rogue Waves in Integrable Turbulence, Phys. Rev. Lett. 114, 143903 (2015).

[106] P. Suret, R. El Koussaifi, A. Tikan, C. Evain, S. Randoux, C. Szwaj, and S. Bielawski, Single-Shot Observation of Optical Rogue Waves in Integrable Turbulence Using Time Microscopy, Nat. Commun. 7, 13136 (2016).

[107] S. Randoux, P. Suret, and G. El, Inverse Scattering Transform Analysis of Rogue Waves Using Local Periodization Procedure, Sci. Rep. 6, 29238 (2016).

[108] D. S. Agafontsev and V. E. Zakharov, Integrable Turbulence and Formation of Rogue Waves, Nonlinearity 28, 2791 (2015).

[109] J. M. Soto-Crespo, N. Devine, and N. Akhmediev, Integrable Turbulence and Rogue Waves: Breathers or Solitons?, Phys. Rev. Lett. 116, 103901 (2016). 